\title{
Endophilin Promotes a Late Step in Endocytosis at Glial Invaginations in Drosophila Photoreceptor Terminals
}

\author{
Ruth Fabian-Fine, ${ }^{1,2 \star}$ Patrik Verstreken, ${ }^{3 *}$ P. Robin Hiesinger, ${ }^{4}$ Jane Anne Horne, ${ }^{2}$ Rita Kostyleva, ${ }^{2}$ Yi Zhou, ${ }^{5}$ \\ Hugo J. Bellen, ${ }^{3,4,5,6}$ and Ian A. Meinertzhagen ${ }^{1,2}$ \\ ${ }^{1}$ Neuroscience Institute, ${ }^{2}$ Department of Psychology, Life Sciences Centre, Dalhousie University, Halifax, Nova Scotia, Canada B3H 4J1, and ${ }^{3}$ Program in \\ Developmental Biology, ${ }^{4}$ Howard Hughes Medical Institute, ${ }^{5}$ Department of Molecular and Human Genetics, and ${ }^{6}$ Division of Neuroscience, Baylor College \\ of Medicine, Houston, Texas 77030
}

Retrieval of synaptic vesicles from the membrane of neurons is crucial to maintain normal rates of neurotransmitter release. Photoreceptor terminals of the fly's eye release neurotransmitter in a tonic manner. They therefore rely heavily on vesicle regeneration. Null mutations in endophilin (endo) block clathrin-mediated endocytosis at the Drosophila neuromuscular junction, where previous analysis of hypomorphic mutations has suggested a function for Endophilin (Endo) before vesicle fission, during membrane bending. Here, at fly photoreceptor synapses, we show that Endo is localized to synaptic vesicles at sites of endocytosis that are glial invaginations called capitate projections, and that when the photoreceptor synapses lack Endo they are impaired in their ability to release neurotransmitter. Detailed ultrastructural analysis of endo null mutant photoreceptor synapses fails to reveal a defect at early stages of vesicle reformation but, instead, reveals an accumulation of clusters of electron-dense, apparently nonfunctional, late endocytotic vesicles. Using dynamin; endo double-mutant photoreceptors, we provide further evidence that ultimately the function of Endophilin is required late in endocytosis, allowing vesicles to progress through the synaptic vesicle cycle.

Key words: clathrin; endophilin; endocytosis; capitate projection; glia; vesicle cycle

\section{Introduction}

In neurons, evidence exists for at least two pathways of synaptic vesicle regeneration (Slepnev and De Camilli, 2000; Jarousse and Kelly, 2001; Kjaerulff et al., 2002; Aravanis et al., 2003; Gandhi and Stevens, 2003). In so-called "kiss-and-run," synaptic vesicles expel their contents but do not collapse into the presynaptic membrane (Ceccarelli et al., 1973; Fesce and Meldolesi, 1999; Verstreken et al., 2002). In clathrin-mediated endocytosis, synaptic vesicles collapse completely in the presynaptic membrane and reform during events that dimple and shape the membrane. Kinetic studies of both pathways in different neuron types suggest that kiss-and-run prevails under low-frequency stimulation, augmented by clathrin-mediated endocytosis during high-frequency stimulation (Ceccarelli et al., 1973; Heuser and Reese, 1973; Palfrey and Artalejo, 1998; Sun et al., 2002; Verstreken et al., 2002). Neurons are also reported to modify their release characteristics and mode of vesicle regeneration under altered stimulation conditions (Zakharenko et al., 2002).

Received July 18, 2003; revised Sept. 26, 2003; accepted 0ct. 3, 2003.

R.F-F. was supported by a postdoctoral fellowship from the Killam Trust of Dalhousie University, P.V. is supported by the Belgian American Educational Foundation, H.J.B. is a Howard Hughes Medical Institute (HHMI) investigator, and P.R.H. was supported by the HHMI and a European Molecular Biology Organization fellowship. I.A.M. was supported by Natural Sciences and Engineering Research Council Genomics Grant GENPJ 239772 and National Institutes of Health Grant EY-03592. We thank the Bloomington Stock Center for flies and Elaine Seto and Tong-Wey Koh for comments. We also thank members of the Meinertzhagen and Bellen labs for valuable discussions.

${ }^{*}$ R.F.-F. and P.V. contributed equally to this work.

Correspondence should be addressed to I. A. Meinertzhagen, Neuroscience Institute, Life Sciences Centre, Dalhousie University, Halifax, Nova Scotia, Canada B3H 4J1. E-mail: iam@dal.ca.

Copyright $\odot 2003$ Society for Neuroscience $\quad$ 0270-6474/03/2310732-13\$15.00/0
The molecular requirements for each of these pathways include the action of the GTPase dynamin, which is required to separate synaptic vesicles attached to the presynaptic membrane and is implicated in both kiss-and-run and clathrin-mediated endocytosis (Artalejo et al., 1995; Verstreken et al., 2002). In contrast, numerous proteins have been proposed to function exclusively in the clathrin-mediated pathway (Zhang and Ramaswami, 1999). Despite evidence for the cooperative action of these proteins, further dissection of their functions in vivo is required to gain a better understanding of how synaptic vesicles form and eventually reenter the functional vesicle pool within an intact nerve terminal.

The formation of new vesicles in clathrin-mediated endocytosis comprises various stages. During the "early stages," the planar membrane bends to form a shallow, hemispherical, clathrincoated pit. Subsequently, in "mid-endocytotic stages," a deeply invaginating coated pit forms, constricted at its neck. Finally during "late stages" of endocytosis, the newly formed vesicle separates from the membrane and is uncoated before reentering the functional vesicle pool. As used in this study, endocytosis is taken to include all of these events, and in vivo and in vitro studies implicate requirements for specific proteins at each of these stages (González-Gaitán and Jäckle, 1997; Cremona et al., 1999; Simpson et al., 1999; Hill et al., 2001; Guichet et al., 2002).

One protein required for clathrin-mediated endocytosis is Endophilin A (Endo), an SH3 domain protein that harbors lysophosphatidic acid acyl transferase (LPAAT) activity (Schmidt et al., 1999; Guichet et al., 2002). By virtue of its enzymatic activity, 
Endo has been proposed to act during early- and midendocytotic stages of endocytosis. By converting lysophosphatidic acid into the more bulky phosphatidic acid, LPAAT has been proposed to induce inward negative membrane curvature to form a new vesicle (Scales and Scheller, 1999; Schmidt et al., 1999). In vivo analysis of endophilin (endo) hypomorphic mutations in Drosophila supports this idea, because mid-stage endocytotic shallow pits accumulate at the membrane of neuromuscular junctions (NMJs) (Guichet et al., 2002). Similarly, injecting Endo-SH3 antibodies into lamprey synapses leads to an accumulation of early and mid-stage endocytotic structures, as well as of some deeply invaginating coated pits (Ringstad et al., 1999). Hence, Endo may aid in the transit from early to mid stages of synaptic vesicle endocytosis.

Here we present evidence that, in Drosophila photoreceptors, Endo plays no obvious role during membrane bending during early endocytosis, but rather that loss of Endo function leads to an accumulation of late endocytotic vesicles. In addition, our analysis reveals that clathrin-mediated endocytosis in Drosophila photoreceptor terminals occurs at specific sites of glial invagination, which possibly couple endocytosis with neurotransmitter reuptake.

\section{Materials and Methods}

Drosophila strains and genetics. For this study, we used the $w$; endo ${ }^{1}$ null allele, the $w$; end $o^{2}$ hypomorphic allele, and the temperature-sensitive $s h i^{t s 1}$ allele (Verstreken et al., 2002). yw; FRT82B endo ${ }^{1}$ was reported previously (Verstreken et al., 2002). yw; eyFLP2 glas-lacZ; FRT82B $w^{+} c l 3 R / T M 3 S b$ was crossed to $y w ; F R T 82 B$ endo ${ }^{1}$ to create flies with eyes homozygous for endo ${ }^{1}$ (Newsome et al., 2000). Expression of wild-type Endo in homozygous mutant endo ${ }^{1}$ eyes was achieved by crossing $y w$; eyGAL4 UAS-FLP; FRT82 GMR-hid 3R CL3R to yw; FRT82B endo ${ }^{1}$ (Stower and Schwarz, 1999). The endo ${ }^{1} \mathrm{P}$-element insertion is an EP P-element with upstream activating sequence (UAS) sites in the correct orientation for expression of wild-type endo (Verstreken et al., 2002). yw $\operatorname{shi}^{\text {tsl }} / Y$; eyFLP5; FRT82B endo ${ }^{1} / F R T 82 B w^{+}$cl3R male flies with doublemutant eyes were generated by crossing $y w$ shitsl $;$ FRT82B $w^{+} c l 3 R / T M 3 S b$ virgins to $y w / Y$; eyFLP5; FRT82B endo ${ }^{1} / T M 3 S b$ males. These two stocks were generated by standard genetics. The control used for experiments performed with homozygous endo ${ }^{1}$ eyes was $y w$ eyFLP2 glas-lacZ; FRT82B $w^{+} c l 3 R /$ FRT82B. For experiments on flies with double-mutant eyes, similar flies at the permissive temperature were used as controls as well as $s h{ }^{t s I}$ flies treated identically to flies with double-mutant eyes (see below).

Immunocytochemistry and adult retina preparation. Immunocytochemistry on adult whole-mounted brains was performed as described (Hiesinger et al., 2001). Anti-Endo (GP69) (Verstreken et al., 2002) was used at 1:500, anti-cysteine string protein (CSP) (Zinsmaier et al., 1994) at 1:200, anti-synaptobrevin (Syb) (Wu et al., 1999) at 1:200, anti-Ras opposite (ROP) (Harrison et al., 1994) at 1:500, and 24B10 (Zipursky et al., 1984) at 1:50. Fluorescent secondary antibodies were from Jackson ImmunoResearch (West Grove, PA) and were used at 1:250. Images were captured with a Zeiss 510 confocal microscope and processed with Amira 2.2 or 2.3 (TGS) and Photoshop 7 (Adobe).

Electrophysiology and phototaxis assay. Electroretinograms (ERGs) from flies immobilized with nail polish (Top Speed, Revlon) were recorded with a fine glass pipette filled with $3 \mathrm{M} \mathrm{NaCl}$ placed on the corneal surface of the fly's eye. The reference electrode was inserted into the thorax. Light flashes of $1 \mathrm{sec}$ were delivered from a $150 \mathrm{~W}$ halogen lamp (Volpi), and field potential recordings were digitized and stored on a PC using Clampex software. Ten flies with mutant eyes and 10 with control eyes were used to record 30 ERG traces, and the amplitudes of the "on" and "off" transients as well as the sustained negative component were analyzed using Clampfit.

A countercurrent phototaxis apparatus (Benzer, 1973) equipped with seven vials was used to determine whether flies exhibited phototaxis. The light source was a $60 \mathrm{~W}$ fluorescent light bulb (GE Lighting). Flies, 10 groups of 20 with mutant eyes and 10 groups of 20 with control eyes ( $h d c$ as a negative control and $y w$ eyFLP; FRT82B $w^{+} c l 3 R / F R T 82 B$ as a positive control) were loaded in the apparatus and allowed to walk seven consecutive times toward light during $10 \mathrm{sec}$.

We should point out that in this work we used the eyFLP technique to create homozygous endo ${ }^{1}$ eyes (Newsome et al., 2000), whereas Rikhy et al. (2002) used a modified version of the EGUFhid technique (Stower and Schwarz, 1999). The technique used by Rikhy et al. (2002) eliminates nonhomozygous mutant cells after differentiation of the cell types in the eye disc (Stower and Schwarz, 1999) and leads to homozygous eyes with disrupted morphology. This roughening may sensitize the genetic background leading to reduced phototaxis reported in Rikhy et al. (2002), whereas we report here that flies with endo ${ }^{1}$ mutant eyes, created with the eyFLP technique, display normal phototaxis.

Fly head fractionation and Western blot analysis. Canton-S fly head homogenates were prepared as described previously (Schulze et al., 1995). Homogenates were fractionated by $0.2 \mathrm{M} / 0.4 \mathrm{M}$ sucrose step centrifugation (Schulze et al., 1995). In Figure 2, "input" was fly head homogenate, "cytosol" was the supernatant material that failed to enter the $0.2 \mathrm{M}$ sucrose layer, and "vesicles" was the material at the interface between the 0.2 and 0.4 M layers (Schulze et al., 1995). Proteins in the fractions were separated by SDS-PAGE and transferred to nitrocellulose. Fifteen micrograms of total protein were loaded per lane. To detect antigens on the blots, primary antibodies were used as follows: anti-Endo 1:5000, anti-Drab3 1:1000 (Schulze et al., 1995), and anti-Syb (R29) 1:5000. Secondary HRP-conjugated antibodies (Jackson ImmunoResearch) were used at 1:5000; bands were visualized by enhanced chemiluminescence (NEN).

Conventional electron microscopy. Twenty-four-hours-old flies with endo ${ }^{l}$ mutant eyes and control flies were fixed under constant Ganzfeld illumination comprising an array of light-emitting diodes within a spherical chamber (Kral and Meinertzhagen, 1989). Fixation was for $2 \mathrm{hr}$ at $23^{\circ} \mathrm{C}$ in $2.5 \%$ of glutaraldehyde and paraformaldehyde in $0.1 \mathrm{M}$ cacodylate buffer, $\mathrm{pH} 7.3$, containing $7 \mathrm{mM} \mathrm{CaCl}_{2}$, followed by postfixation in $2 \% \mathrm{OsO}_{4}\left(1 \mathrm{hr}\right.$ at $\left.4^{\circ} \mathrm{C}\right)$, all as described previously (Meinertzhagen, 1996). Preparations were examined at 60 or $80 \mathrm{kV}$ using Philips 201C or Tecnai 12 electron microscopes.

Postembedding immunogold labeling for electron microscopy. Canton-S flies were dissected in $0.1 \mathrm{~m}$ phosphate buffer (PB), pH 7.4, with $4 \%$ paraformaldehyde, $0.05 \%$ glutaraldehyde, and $0.2 \%$ picric acid (Somogyi and Takagi, 1982), soaked in PB for $15 \mathrm{~min}$, and then immersed in $0.125 \mathrm{M}$ triethanolamine hydrochloride in $\mathrm{PB}$ for $30 \mathrm{~min}$ to quench unreacted aldehydes. Heads were cryoprotected for $>2 \mathrm{hr}$ in increasing concentrations of glycerol in $\mathrm{PB}$ up to $30 \%$ and then impact frozen on a polished copper mirror at $-193^{\circ} \mathrm{C}$ (Leica MM80) and, using a Leica automatic freeze-substitution system (AFS), freeze-substituted in methanol with $0.5 \%$ uranyl acetate for $24 \mathrm{hr}$ at $-85^{\circ} \mathrm{C}$. Specimens were subsequently rinsed in methanol, and the temperature was then raised to $-50^{\circ} \mathrm{C}$ before infiltration and embedment in Lowicryl HM 20 (Agar Scientific) as described previously (Fabian-Fine et al., 2001). The resin was polymerized at $-50^{\circ} \mathrm{C}$ using UV light, and ultrathin sections $(50 \mathrm{~nm})$ were collected on Pioloform-coated single-slot nickel grids. For uniform treatment, all sections were mounted on grids using a support plate (Leica Microsystems). Sections were soaked in PB for 30 min and incubated in blocking medium (BM) (1\% BSA, $10 \%$ fetal calf serum in $\mathrm{PB})$ for $30 \mathrm{~min}$ at $23^{\circ} \mathrm{C}$. Anti-Endo (GP69) was used at $1: 100$ overnight at $4^{\circ} \mathrm{C}$, $1 \mathrm{hr}$ at $37^{\circ} \mathrm{C}$, and overnight again at $4^{\circ} \mathrm{C}$. Polyclonal anti-clathrin (Sigma, St. Louis, MO) was used at 1:50 overnight at $4^{\circ} \mathrm{C}$. Sections were then rinsed thoroughly in $\mathrm{PB}$ and incubated in $\mathrm{BM}$ at $37^{\circ} \mathrm{C}$ for $30 \mathrm{~min}$. Secondary antibodies conjugated to $10 \mathrm{~nm}$ gold particles (Jackson ImmunoResearch) applied for $4 \mathrm{hr}$ at $37^{\circ} \mathrm{C}$ were used at 1:100 for Endophilin labeling and at 1:50 for clathrin labeling. Preparations were washed in $\mathrm{BM}$, in PB, and finally in double-distilled water. Sections were contrasted with uranyl acetate $(4 \mathrm{~min})$ and Reynold's lead citrate $(2 \mathrm{~min})$ as described previously (Meinertzhagen, 1996).

Specificity of labeling for Endo was confirmed by the absence of immunolabeling in preparations from which primary anti-Endo was omitted. The specificity of anti-Endo (GP69) was demonstrated previously (Verstreken et al., 2002). The specificity of anti-clathrin (Sigma) was tested by Western blot analysis and labeling of tissue. At the NMJ, label- 
ing with this anti-clathrin antibody reveals presynaptic enrichment (Zhang et al., 1998). To demonstrate the specificity of this antibody more directly, anti-clathrin antibodies were first preadsorbed overnight at $4^{\circ} \mathrm{C}$ against a 178 $\mathrm{kDa}$ protein corresponding to clathrin heavy chain, from fly head extract. Subsequently control preparations were incubated with this preabsorbed antibody. Specific labeling was absent in these preparations.

Quantitative analysis of electron microscopic images. We made single-blind counts of synaptic organelles in single-section profiles of photoreceptor terminals in all genotypes examined (Meinertzhagen and O'Neil, 1991). We next counted the following organelle profiles: $30 \mathrm{~nm}$ synaptic vesicles, T-shaped presynaptic ribbons at tetrad synapses, and capitate projections. Using previous criteria (Pyza and Meinertzhagen, 1997; Rybak and Meinertzhagen, 1997), the latter were counted as shallow (with a head invaginating not more than half its diameter), single (penetrating, with a single head profile; see Fig. $7 E$ ), and multiple (penetrating, with more than one head; see Fig. $7 F$ ). The perimeters and cross-sectional areas of terminal profiles were measured with software (NIH Image). Tests for statistical significance in organelle counts were first made by an unweighted means ANOVA, followed by a Tukey honestly significant difference (HSD) test, using software (Systat 5.2.1).

We evaluated the distribution of 207 (anticlathrin) and 89 (anti-Endophilin) immunogold particles from 10 and 16 micrographs, respectively, containing both particle clusters and wide areas with only occasional particles. We counted particles in two categories, either associated with membrane, for which we designated a border of $60 \mathrm{~nm}$, two synaptic vesicle diameters (Meinertzhagen and O'Neil, 1991), or in the cytoplasm. Membrane-associated particles were either close to capitate projections or to the plasmalemma. Those in the cytoplasm occurred at lower densities, which we calculated from measurements of the membrane perimeter and cross-sectional area of the photoreceptor terminals. The correct attribution of particles to capitate projection or plasmalemma was made difficult by the complex geometry of the former, because in any one section capitate projection heads are rarely connected to the plasmalemma by a stalk. Thus some plasmalemma-associated particles probably belong to capitate projection stalks lying just in front of or behind the section plane. To test the significance of differences in distribution, we used an unweighted means ANOVA, followed by a Tukey HSD test, as above. Comparisons were made on the subcounts of particles in each category made on individual micrographs.

Synaptic vesicle depletion-recovery assay. The functional vesicle pool in endo ${ }^{1}$ mutant eyes was depleted by exposing flies with double-mutant eyes $\left(s h i^{t s l} ; e n d o^{l}\right)$ to the restrictive temperature $\left(29^{\circ} \mathrm{C}\right)$ for $s h i^{t s l}$ to block synaptic vesicle recovery. Returning flies to the permissive temperature $\left(18^{\circ} \mathrm{C}\right)$ restores the function of dynamin but not Endo. We allowed flies to recover for various times at the permissive temperature and then processed their eyes for electron microscopy (EM) (see Fig. 8A).

We performed the following set of experiments on flies with $s h i^{t s l}$; endo ${ }^{1}$ eyes as well as on $s h i^{t s l}$ flies (as a control). Flies were exposed for 15 min to $29^{\circ} \mathrm{C}$ in normal room light and then (1) fixed at $29^{\circ} \mathrm{C}$ before
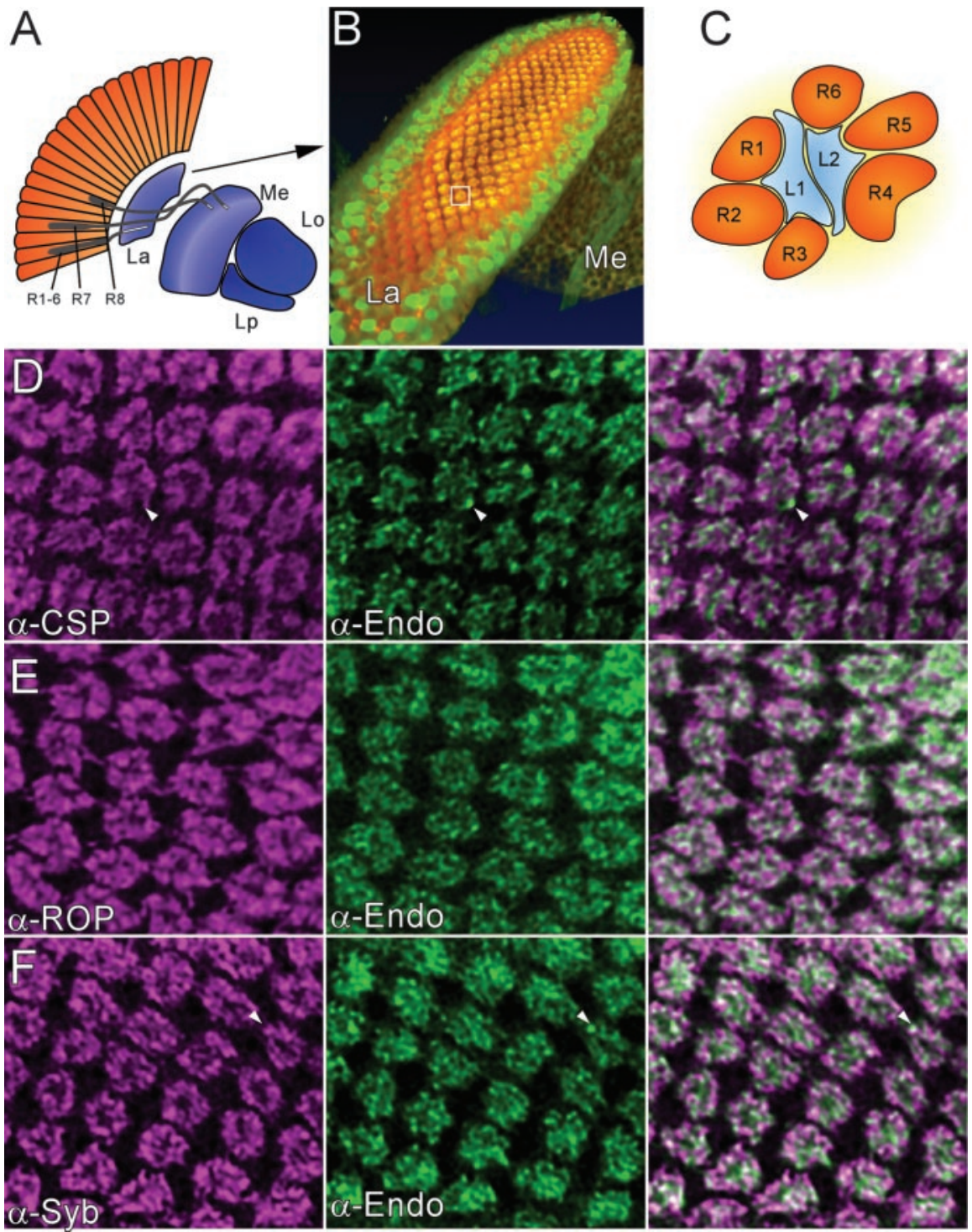

Figure 1. Localization of Endo in photoreceptor terminals. A, Schematic representation of the optic lobe neuropiles in DrosophIla. R, Photoreceptor cell; La, lamina; Me, medulla; Lo, lobula; Lp, lobular plate. An array of photoreceptors (gray) project axons that anti-Endophilin (green) and anti-Synaptobrevin (red). Cartridges comprising R1-R6 terminals that innervate lamina monopolar re immunoreactive for both Endo and Syb (overlap: orange/yellow). Note, however, that Endo is also present in unidentified erminals (R1-R6; orange), surrounding lamina monopolar cells L1 and L2 (blue). In endo ${ }^{7}$ mutant eyes created by the ey-FLP (me, almost all R1-R6 are mutant. $D-F$, Colocalization of synaptic markers (magenta) with Endo in lamina cartridges, show as single confocal images from deconvolved three-dimensional datasets. D, CSP and Endo extensively colocalize; however, Endopositive (SP negative puncta exist (arrowhead). E, Double-labeling for ROP and Endo; $F$, double-labeling for Syb and Endo. Endo and Syb overlap, although not entirely $(F$, arrowhead).

processing for EM, (2) shifted to $18^{\circ} \mathrm{C}$ for an additional $15 \mathrm{~min}$ and finally fixed at $18^{\circ} \mathrm{C}$ for $\mathrm{EM}$, and (3) shifted to $18^{\circ} \mathrm{C}$ for $30 \mathrm{~min}$ and finally fixed at $18^{\circ} \mathrm{C}$ for EM. As another control, (4) $\operatorname{sh}^{i s}{ }^{t}$;endo ${ }^{1}$ flies were held at $18^{\circ} \mathrm{C}$ and fixed at $18^{\circ} \mathrm{C}$ for EM.

\section{Results}

Endophilin is present in photoreceptor terminals

To study the role of Endo in endocytosis we have used the photoreceptors of the fly's eye. The Drosophila eye consists of $\sim 800$ ommatidia, each containing eight photoreceptors, R1-R8. Six of these, R1-R6, emit axons that terminate and synapse in the first optic neuropile, the lamina (Fig. 1A). The axons of R7 and R8 synapse in the second optic neuropile, the medulla. In the lamina, 


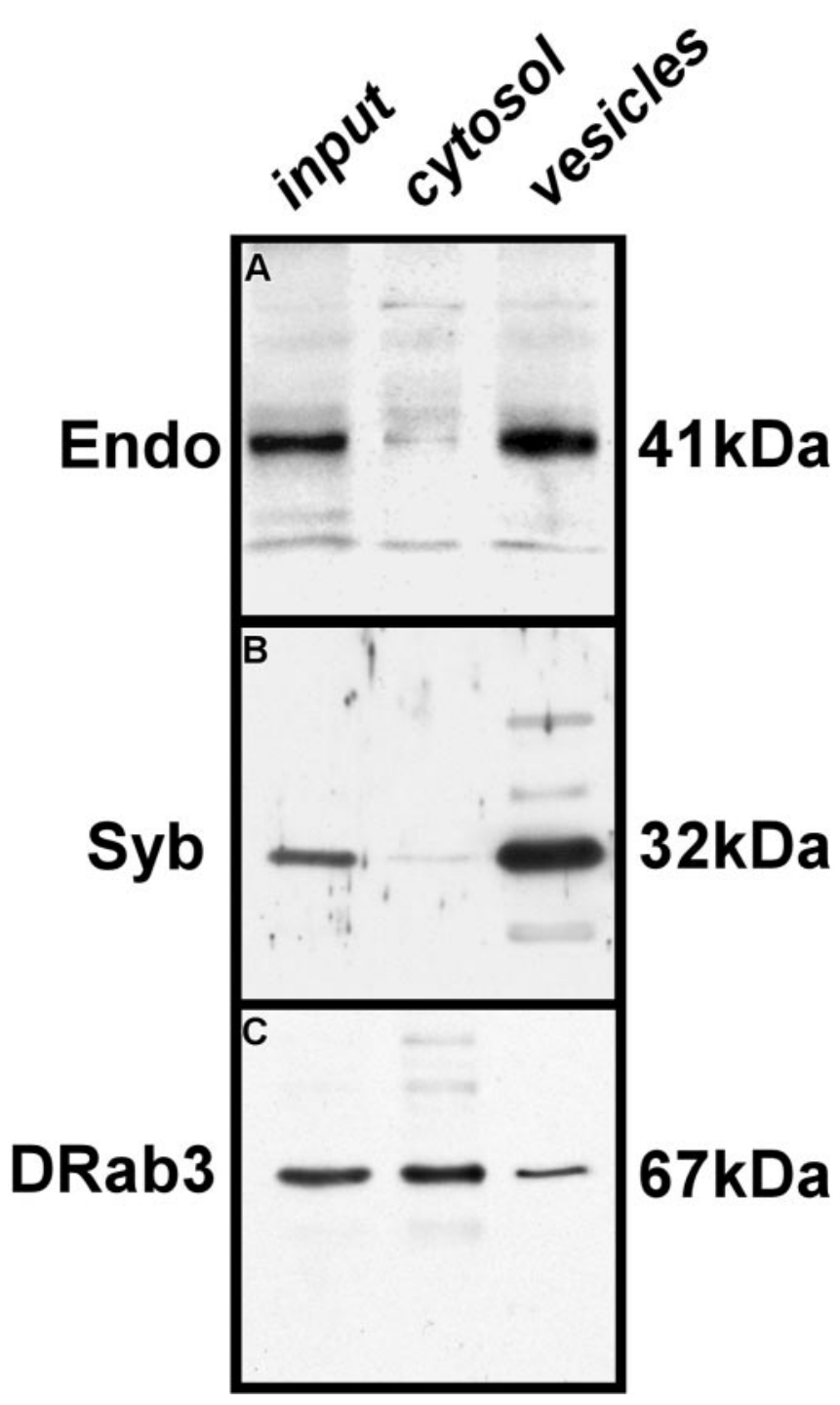

Figure 2. Subcellular distribution of Endo. A-C, Protein extracts collected from various subcellular fractions from fly heads (see Materials and Methods) were resolved on polyacrylamide gels and transferred to nitrocellulose and probed for the presence of Endo $(A)$, Syb $(B)$, and DRab3 ( $C$. Details on fractions loaded in lanes 1-3 (input, cytosol, vesicles) are in Materials and Methods. Endo appears in the vesicle and membrane fraction but is almost absent from the cytosolic fraction.

the terminals of photoreceptors R1-R6 sort precisely into lamina cartridges (Fig. $1 B$ ) so that each cartridge receives six terminals that originate from different ommatidia (Fig. 1C) (Braitenberg, 1967). Each cartridge is surrounded by three epithelial glial cells (Meinertzhagen and O'Neil, 1991); hence the fly's eye harbors a highly organized set of synaptic contacts allowing us to assess readily morphological and ultrastructural defects in mutant photoreceptor terminals.

To determine whether Endo is present in the terminals of R1-R6, we dissected laminas from control flies and labeled them with anti-Endo (Verstreken et al., 2002) and monoclonal antibody ( $\mathrm{mAb}) 24 \mathrm{~B} 10$, which is specific to photoreceptor cell membranes (Zipursky et al., 1984). Double labeling with 24B10 and Endo antibodies indicated that Endo colocalized extensively with 24B10-positive regions and is associated with lamina cartridges (data not shown), suggesting that Endo is present at photoreceptor terminals.

To determine more precisely the localization of Endo within
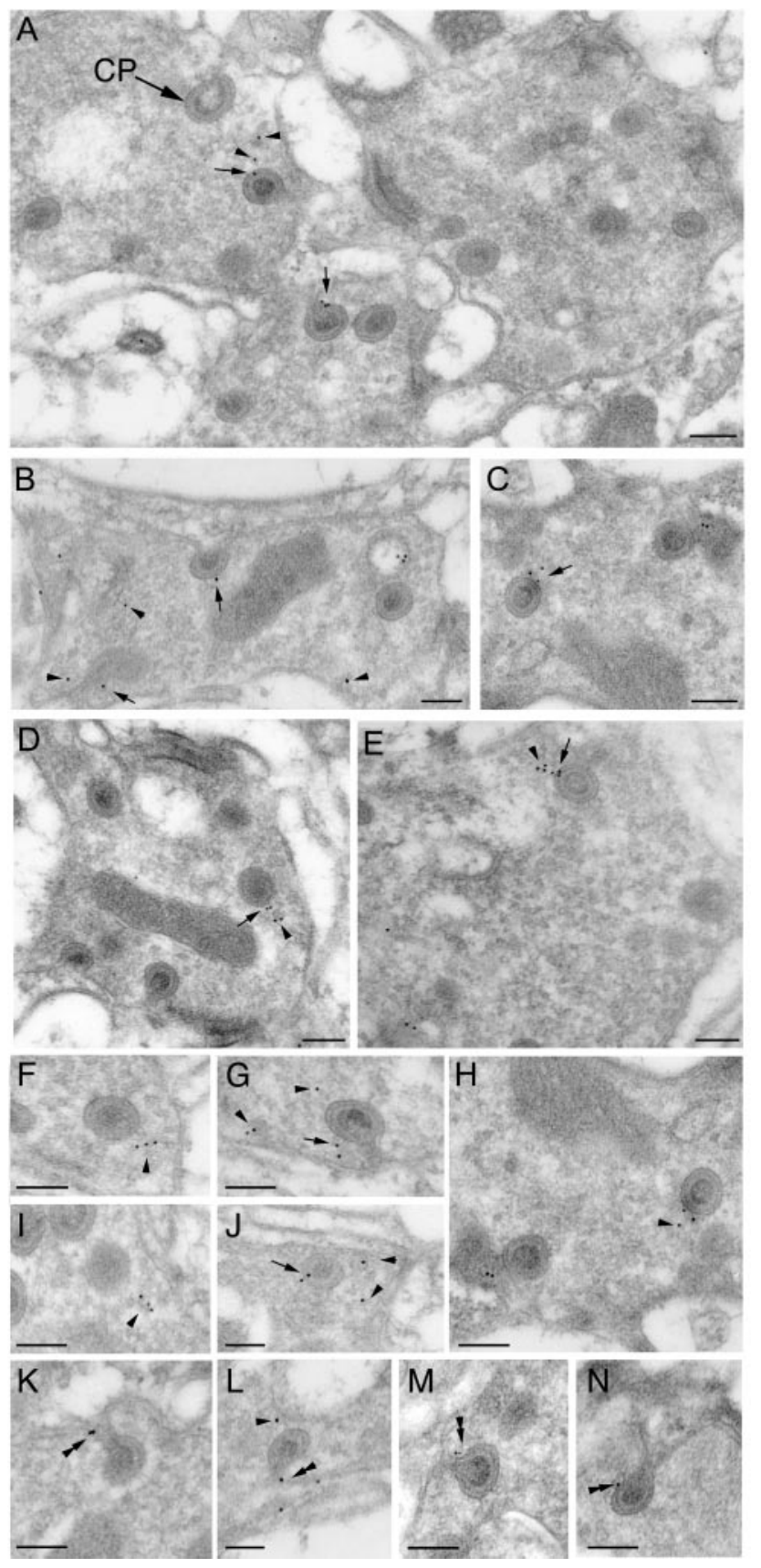

Figure 3. EM immunogold labeling of Endo in wild-type photoreceptor terminals. Immunogold particles labeling Endo localize to capitate projections, invaginations from the surrounding epithelial glial cells. $A-J$, Gold particles marked by arrows are located at the heads of capitate projections $(A, C P)$. Particles marked by arrowheads are on vesicles close to capitate projections. $K-N$, Particles are also detected along the penetrating shafts of capitate projections (double arrowhead). Scale bars, $0.2 \mu \mathrm{m}$.

the lamina cartridges, we double labeled laminas with anti-Endo and various synaptic markers. Confocal stacks of such immunolabeled preparations were scanned, and the three-dimensional datasets were deconvolved using previously established algorithms (Hiesinger et al., 2001). Endophilin was enriched in lamina cartridges and, along with synaptic markers such as the vesicle-associated proteins neuronal Syb (Fig. $1 B, F$ ) (Wu et al., 1999) and CSP (Fig. 1D) (Zinsmaier et al., 1994), localized to the photoreceptor synaptic terminals. Endo did not entirely colocalize with CSP and Syb and was also found in compartments within 
the terminal that were devoid of either of these vesicle markers (Fig. 1). Likewise, Endo mostly colocalized with ROP, a cytosolic protein at the nerve terminal (Fig. 1E) (Harrison et al., 1994). Hence, these data suggest that Endo is localized to specific compartments within the photoreceptor terminals.

\section{Endophilin is present on synaptic vesicles}

To further assess the subcellular localization of Endo, we fractionated fly heads to enrich the synaptic vesicle and cytosolic proteins (Schulze et al., 1995). The fractions were separated by SDS-PAGE and transferred to nitrocellulose. To test the purity of the fractions, we probed the blots with antibodies that recognize various neuronal markers (Fig. $2 A-C$ ). Immunoreactivity to the synaptic vesicle marker Syb (Deitcher et al., 1998) was found in the synaptic vesicle-enriched fraction but not in the cytosolic fraction (Fig. $2 B$ ), whereas the synaptic vesicle cycle regulatory protein DRab3 (Schulze et al., 1995) was detected both in the cytosol fraction and in the vesicle-enriched fractions (Fig. 2C). When a blot was probed with anti-Endo antibody, an antigen at $41 \mathrm{kDa}$ was recognized (Fig. $2 \mathrm{~A}$, input, lane 1 ) that corresponded to the predicted molecular weight of EndoA (Guichet et al., 2002; Rikhy et al., 2002; Verstreken et al., 2002). Endo was present in the vesicle-enriched fraction (Fig. 2, vesicles, lane 3) and was not prominent in the cytosolic fraction (Fig. 2, cytosol, lane 2). The synaptic vesicle-enriched fraction probably contained some membrane contaminants (Schulze et al., 1995), so that although we cannot exclude Endo as having some membrane association, our data nevertheless indicate that at the synapse Endo is associated with vesicles.

\section{Endo and clathrin colocalize near sites of glial invagination}

To determine the ultrastructural localization of Endo within photoreceptor terminals in the lamina, we performed immunogold labeling (Fig. 3). Localization of Endo within the presynaptic photoreceptor terminal profiles was confined to areas on or near sites at which the photoreceptor synaptic terminals are invaginated by epithelial glial cells (Fig. 3). Such invaginations form characteristic stalked organelles called capitate projections (Fig. $3 A$ ), but they are still of unknown function (Trujillo-Cenóz, 1965; Stark and Carlson, 1986). Endo labeling was detected near the heads of the capitate projections (Fig. 3A-I), possibly associated with synaptic vesicles, and was present at the membrane of the capitate projection (Fig. $3 A-I$ ), where it was particularly convincing at the actual head (Fig. $3 E-G$ ). Label was seen at the heads of both types of capitate projection head: shallow (Fig. 3B) and invaginating (Fig. 3C). Finally, gold labeling was detected along the shaft of the capitate projection (Fig. $3 K-N$ ) as well as close to the surrounding plasma membrane (Fig. $3 A$ ). The distribution of gold particles was assessed from their density over or near the capitate projections, the plasma membrane, and the cytoplasm (see Materials and Methods). The density of particles was $21.9 \pm$ $18.0 / \mu \mathrm{m}^{2}$ for the capitate projections, $8.8 \pm 6.2 / \mu \mathrm{m}^{2}$ for the plasma membrane, and $1.85 . \pm 1.43 / \mu \mathrm{m}^{2}$ for the cytoplasm (mean $\pm \mathrm{SD} ; n=16$ ). The density over the capitate projections was significantly greater than that over the membrane $(p=$ 0.0114 ) and the cytoplasm ( $p=0.0013$ ). Hence, in photoreceptor terminals, our data from both fractionation assays and immunogold labeling indicate that Endo is localized primarily to synaptic vesicles that are close to capitate projections or localized to the membrane covering the capitate projections themselves.

Endophilin is essential for clathrin-mediated endocytosis (Ringstad et al., 1999; Gad et al., 2000; Verstreken et al., 2002). To
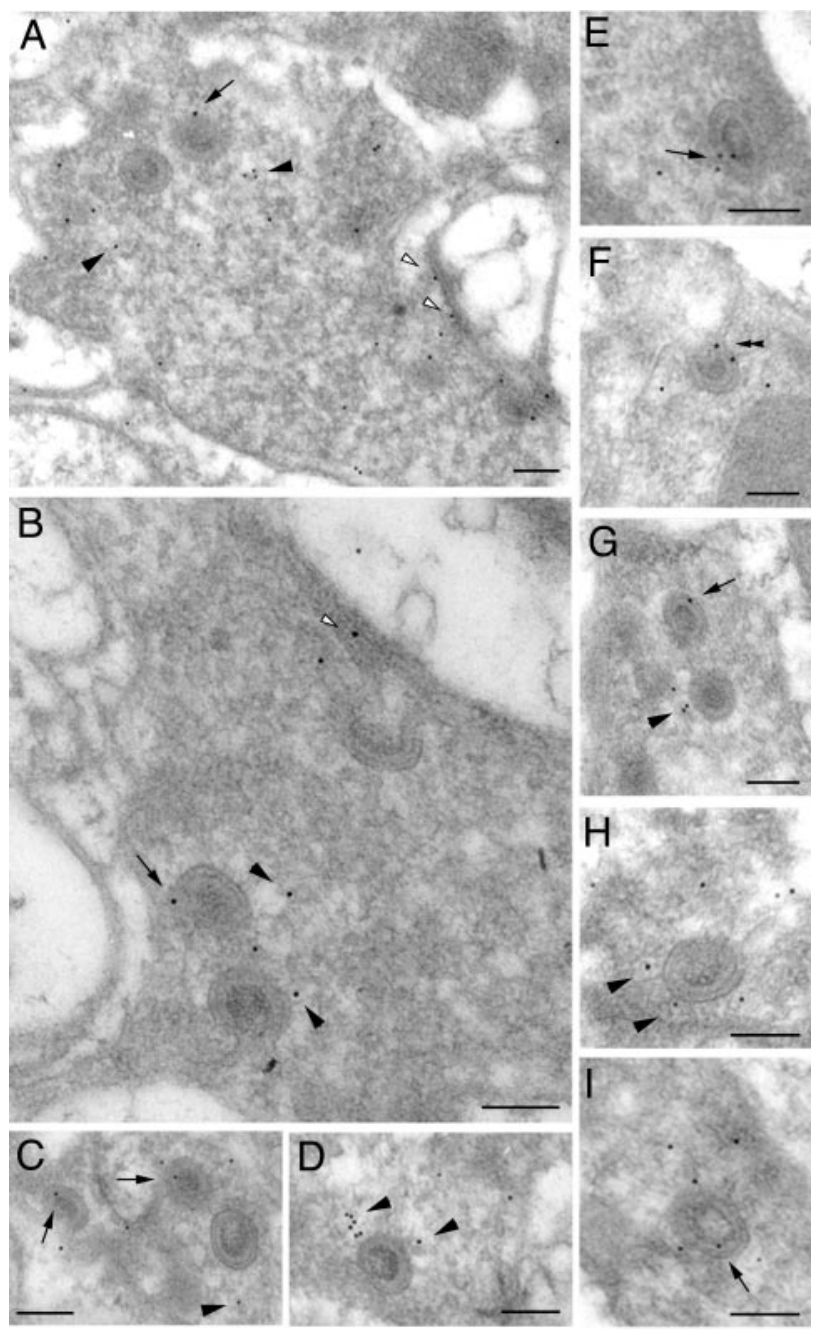

Figure 4. EM immunogold labeling of clathrin heavy chain in wild-type photoreceptor terminals. A-l, Anti-clathrin immunogold particles often distribute close to capitate projections (arrowheads). Particles are also found in association with capitate projections themselves (arrows), and some also localize to the plasma membrane ( $A, B$, open arrowheads). Scale bars, $0.2 \mu \mathrm{m}$.

correlate the ultrastructural localization of Endo with that of clathrin in photoreceptor terminals, we also immunolabeled lamina ultrathin sections with anti-clathrin heavy-chain antibodies (Fig. 4). Interestingly, labeling with anti-clathrin antibodies showed a pattern similar to that of Endo. clathrin labeling was apparent at and near capitate projections (Fig. 4A-I); however, unlike Endo, clathrin immunolabeling was more widespread in the terminal and more prominent along the plasma membrane of the photoreceptor terminal profile (Fig. $4 A, B$ ). The distribution of gold particles assessed as for anti-clathrin immunolabeling revealed that the density of particles was $62 \pm 25.5 / \mu \mathrm{m}^{2}$ for the capitate projections, $17.7 \pm 13.7 / \mu \mathrm{m}^{2}$ for the plasma membrane, and $11.0 \pm 5.6 / \mu \mathrm{m}^{2}$ for the cytoplasm (mean $\pm \mathrm{SD} ; n=10$ ). The density over the capitate projections was significantly greater than the other two $(p<0.001)$. The localization of these two endocytotic markers at sites of glial invagination into capitate projections suggests a role for capitate projections in clathrinmediated retrieval of synaptic vesicles and indicates the existence of specific endocytotic zones in the photoreceptor terminals of Drosophila. 
endo mutations impair the synaptic function of photoreceptors

endo null mutations cause homozygous lethality. To study the function of Endo in the adult fly photoreceptor cells, we have used the eyFLP technique (Newsome et al., 2000) to create homozygous mutant endo ${ }^{1}$ photoreceptor cells in heterozygous flies. Eyes that lack Endo develop normally (Fig. $5 A, B$ ). Sections through the retina show a normal organization of the rhabdomeres, the photosensitive organelles in Drosophila photoreceptors (Fig. $5 C, D$ ). These data demonstrate that Endo A, unlike other proteins involved in clathrin-mediated endocytosis, such as $\alpha$-adaptin (Berdnik et al., 2002; P. Verstreken and H. J. Bellen, unpublished observations) or dynamin (Poodry et al., 1973), is not required for development of the Drosophila eye.

Within their cartridges in the lamina, the terminals of R1-R6 provide synaptic input to a set of lamina cells, in particular the large lamina monopolar cells L1 and L2, which they contact in a stereotyped manner (Meinertzhagen and O'Neil, 1991). The axons of photoreceptors R7 and R8, on the other hand, project to the distal layers of the medulla (Fischbach and Dittrich, 1989) (Fig. $1 A-C$ ). To investigate whether Endophilin is involved in axon growth and pathfinding, target recognition, or gross synaptic development, we have labeled photoreceptor cell membranes with $\mathrm{mAb} 24 \mathrm{~B} 10$, a photoreceptor-specific membrane marker, and visualized the axon projection patterns and synaptic contacts in the lamina and medulla of control and endo ${ }^{1}$ mutant eyes. Mutant R8 and R7 photoreceptors form normal synapses in the correct layers of the medulla (Fig. 5E,F). Mutant R1-R6 also form morphologically normal synaptic contacts in the lamina. To determine whether the sorting of the photoreceptors R1-R6 into lamina cartridges is normal in endo ${ }^{1}$ mutants, we counted the number of their terminals per cartridge in EM images of control and endo ${ }^{1}$ mutant laminas. We did not find a difference in the number of terminals per cartridge in endo ${ }^{1}$ mutant eyes compared with controls (Fig. 5G,H). Hence, Endo is not involved in axon pathfinding or in growth cone target recognition in the visual system of Drosophila.

Previous work has shown that at the NMJ of endo null larvae $\left(e n d o^{1}\right.$ and $e n d o^{\Delta 4}$ ), synaptic function is impaired (Verstreken et al., 2002). We tested whether Endophilin is also involved in the synaptic vesicle cycle in photoreceptors by recording ERGs from control and endo ${ }^{1}$ mutant eyes and by testing the ability of the fly to exhibit phototaxis. The ERG records the light-evoked depolarization and subsequent hyperpolarization of the photoreceptor membrane as a sustained negative response (Heisenberg, 1971), thus indicating the presence of a normal phototransduction cascade. In addition, at the beginning and end of the light flash, the on and off transients of the ERG also report the responses of postsynaptic cells during photoreceptor neurotransmission in the lamina that have been attributed to the main targets of R1R6, the monopolar lamina cells L1 and L2 (Coombe, 1986). In response to a light flash, the photoreceptors in control and endo ${ }^{1}$ mutant eyes depolarize in a similar manner; however, the on and off transients are severely reduced or absent in endo ${ }^{1}$ mutants (Fig. 6A,B), in agreement with data reported by Rikhy et al. (2002). This defect is specific to endo because expression of wildtype endo in endo ${ }^{1}$ mutant photoreceptors leads to complete restoration of the on and off transients (Fig. 6A, EGUF; FRT82B endo ${ }^{1}$ ). The fact that several mutations that impair synaptic endocytosis in the Drosophila eye also lack light-evoked on and off transients, for example stn (Homyk and Pye, 1989), shi (Kelly, 1974; Chen and Stark, 1993; Sanyal et al., 2001), and syt (Littleton et al., 2001), is consistent with the ERG defect in endo ${ }^{1}$ mutants
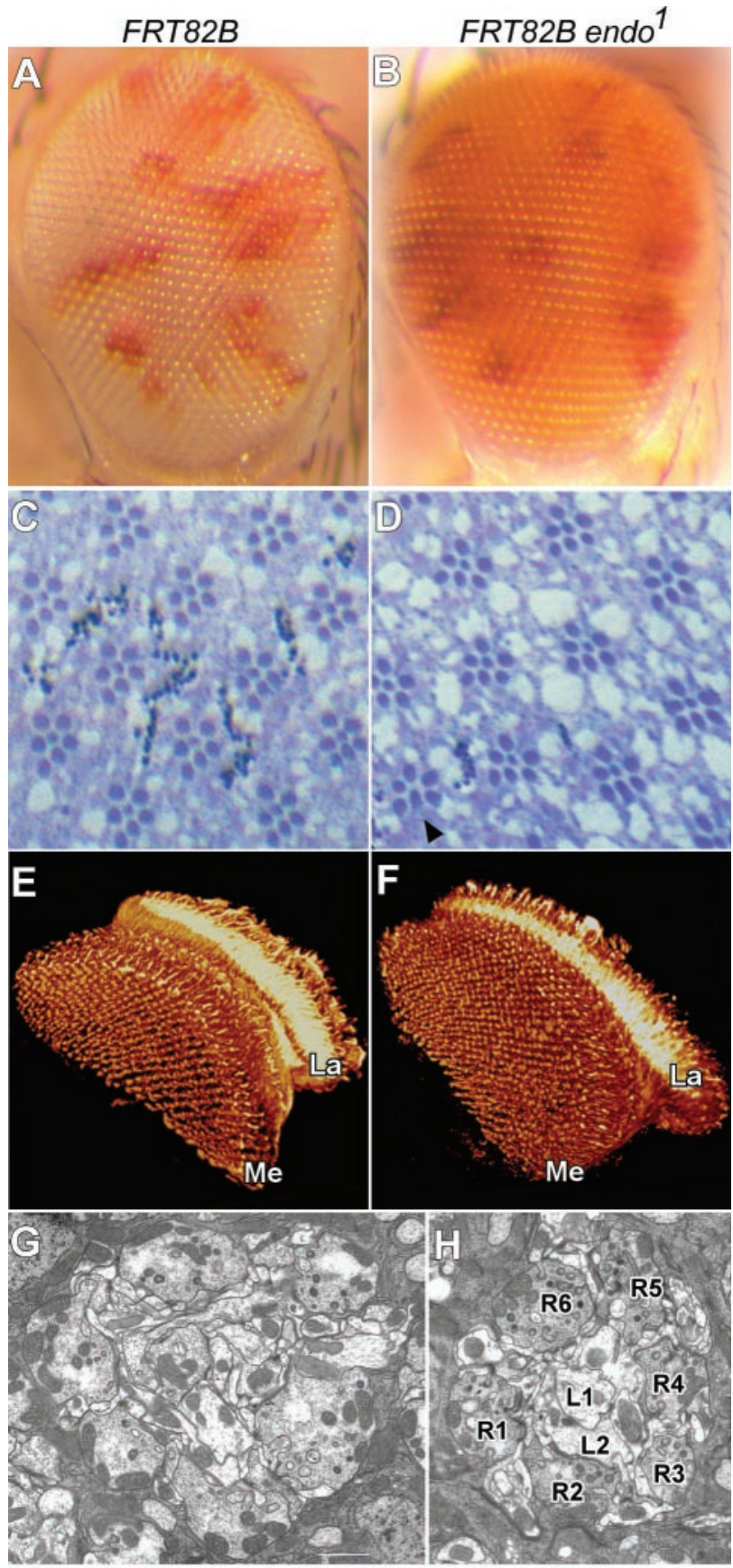

Figure 5. Endo does not affect eye development. $A, B$, External morphology of compound eyes that are almost entirely homozygous for either a control chromosome arm $(A)$ or mutant for endo ${ }^{1}(B)$. Control yw eyFLP; FRT82B/FRT82B $C l w^{+}(A)$ and endo ${ }^{7}$ mutant $y w$ eyFLP; FRT82B endo ${ }^{1} / F R T 82 B C l w^{+}(B)$ have an indistinguishable external appearance to their eyes. Red patches are remaining heterozygous or homozygous nonmutant tissue, whereas homozygous endo ${ }^{1}$ eye tissue is white. $C, D$, Semithin plastic sections through the retinas of control $(C)$ and endo ${ }^{7}$ mutant $(D)$ eyes show normal numbers of photoreceptors in each ommatidium in mutant eyes. Six outer rhabdomeres and one of the two central rhabdomeres are visible in each ommatidial cross section. Rarely a photoreceptor is missing from ommatidia in endo ${ }^{7}$ mutant eyes $(D$, arrowhead). $E$, $F$, Projection patterns of the $\mathrm{R} 7$ and $\mathrm{R} 8$ control $(E)$ and endo ${ }^{1}$ mutant ( $F$ ) photoreceptors into the optic lobe revealed by $24 \mathrm{~B} 10$. The terminals of $R 7$ and $R 8$ beneath endo ${ }^{7}$ mutant eyes $(F)$ appear as ordered as those beneath control $(E)$ and wild-type (Hiesinger et al., 1999) eyes. La, Lamina; Me, medulla. G, H, EM cross-sections of control ( $G$ ) and endo mutant $(H)$ lamina cartridges. The organization of the mutant cartridge shows no obvious differences from that of a control cartridge. The profiles of R1-R6 as well as of their chief lamina cell targets, $\mathrm{L} 1$ and $\mathrm{L} 2$, are indicated in $H$. Scale bar, $1 \mu \mathrm{m}$. 
also arising from a defect in the synaptic vesicle cycle, likely synaptic vesicle endocytosis. On the other hand, the transients of the ERGs not only result from lightevoked transmission in the lamina, but should also reflect the synchrony of that transmission; it is not clear how reliably the transients can be expected to reveal greatly reduced or desynchronized transmission, especially in mutants such as endo ${ }^{1}$ from which the complete lack of transients is not invariable.

To test whether flies with endo ${ }^{1}$ mutant eyes have normal vision, we examined phototaxis using a countercurrent phototaxis assay (Benzer, 1973). Flies are loaded into a tube with a light source on one side and allowed to walk toward the light for 10 sec. Flies that exhibited positive phototaxis were separated from those that did not, and all flies were retested in seven consecutive trials. In this assay, control flies preferentially walk toward the light (Fig. 6C); however, flies that lack histidine decarboxylase and are therefore deficient in the production of histamine (Burg et al., 1993), a neurotransmitter of fly photoreceptors (Hardie, 1987), fail to exhibit normal phototaxis (Fig. 6C). Flies with endo ${ }^{1}$ mutant eyes behave like control flies in this phototaxis assay. A phototaxis assay reported by Rikhy et al. (2002) also on flies with endo ${ }^{1}$ mutant eyes showed, by contrast, that endo ${ }^{l}$ partially impairs phototaxic behavior. Although both studies use the Benzer (1973) countercurrent assay method, there are many reasons why the conditions of the two assays could have differed, for example in the light intensity chosen. Furthermore, the genetic background differs between our flies (see Materials and Methods). Moreover, we report the number of individual positive phototaxis choices, whereas Rikhy et al. (2002) report a single combined phototaxis index. Even so, our data and those of Rikhy et al. (2002) suggest that despite the almost complete lack of on and off transients, neurotransmitter release in tonically active photoreceptors is not blocked totally (Fig. 6A), in agreement with previous findings at the NMJ (Verstreken et al., 2002).

\section{Synaptic vesicles in endo mutant terminals cluster and appear darker than in nonmutant controls}

To study the function of Endo in greater detail, we investigated the synaptic ultrastructure of R1-R6 photoreceptor terminals in the lamina by EM and quantified several features (Table 1). In endo ${ }^{1}$ eyes, homozygous mutant photoreceptors innervate lamina cells to form a cartridge of normal composition (Fig. 5G,H). Synaptic vesicles in control nonmutant terminals of R1-R6 were pale and dispersed (Fig. $7 A, B$, Table 1). Compared with these controls, the most striking feature of mutant photoreceptors was

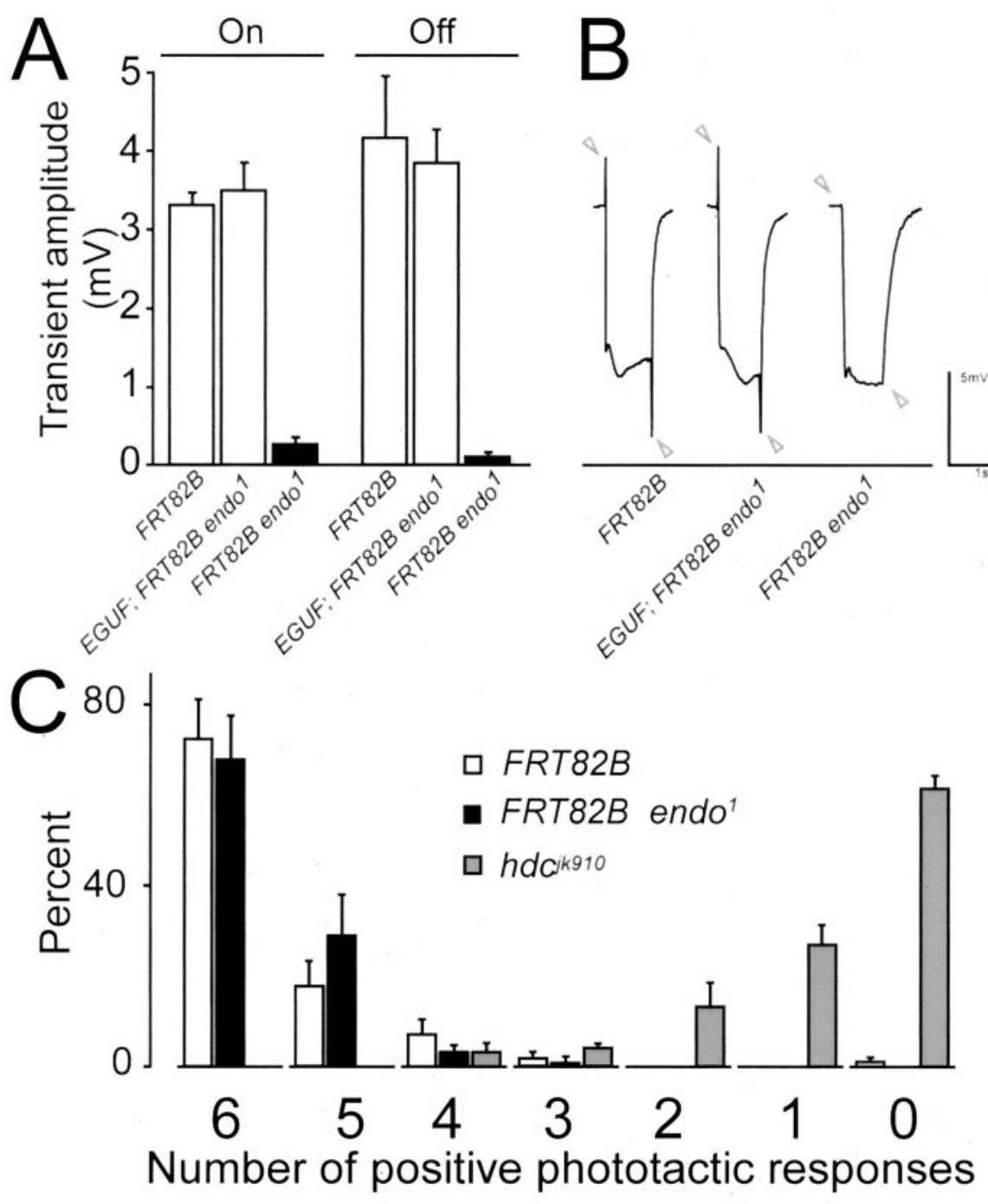

Figure 6. Endo is required for normal synaptic function of Drosophila photoreceptors but not for vision. $A, B$, ERGs recorded from flies with control eyes ( $y w$ eyFLP; FRT82B/FRT82B $d w^{+}$labeled "FRT82B") show a normal sustained negative component ( $\sim 10$ $\mathrm{mV}$ ) in response to a light flash and transients at lights on and lights off, respectively ( $B$, open arrowheads; first recording in the ( $w^{+}$labeled "EGUF; FRT82B endo" "), on and off transients are present, as normal ( $B$, open arrowheads, second recording in the right panel). Expression of wild-type Endo is achieved by expression of GAL4 from the eye promoter (EGUF). This GAL4 can bind to the recording of the right panel in $B$, however, ERGs from endo mutant eyes ( $y w$ eyFLP; FRT82B endo ${ }^{1} / F R T 82 B$ cl $w^{+}$labeled "FRT82B endo ${ }^{1 "}$ ) either have severely reduced on and off transients in a few recordings or lack them in most others. The light-evoked normal. Amplitudes for the ERG transients shown in $A, n=10$ for each genotype, show statistically significant differences ( $p<$ 0.001 ; $t$ test). C, Countercurrent phototaxis assays performed on control FRT82B flies, on $h d c^{J K 10}$ flies lacking neurotransmitter and on flies with endo ${ }^{1}$ mutant eyes. Values reveal the mean proportion of positive phototaxis in five groups each of $20 \mathrm{flies}$ for each genotype. Flies with endo $0^{1}$ mutant eyes exhibit phototaxis identical to controls, unlike flies that lack neurotransmitter $\left(h d c^{J K 910}\right)$. (Error bars represent SEM; $n=5$.)

that synaptic vesicles in endo ${ }^{1}$ terminals appeared more electron dense on their surface (Fig. 7C,D), were mostly found in clusters (Fig. 7C-F), and appeared to be spaced more closely within the clusters. These features were all borne out quantitatively (Fig. $7 G, H)$ and were visible to naive observers. To be confident that clustered vesicles in mutant terminals did not appear to have an increased electron density through a simple overall difference in the contrast of the micrograph, we normalized their density to that of vesicles at the active zone (Fig. $7 G$ ). Interestingly, such vesicle clusters in endo mutant photoreceptor terminals appeared 
Table 1. Organelle counts in single endo ${ }^{1}$ and double-mutant $\operatorname{sh}^{\text {ts }}{ }^{1}$;endo ${ }^{1}$ photoreceptor terminals

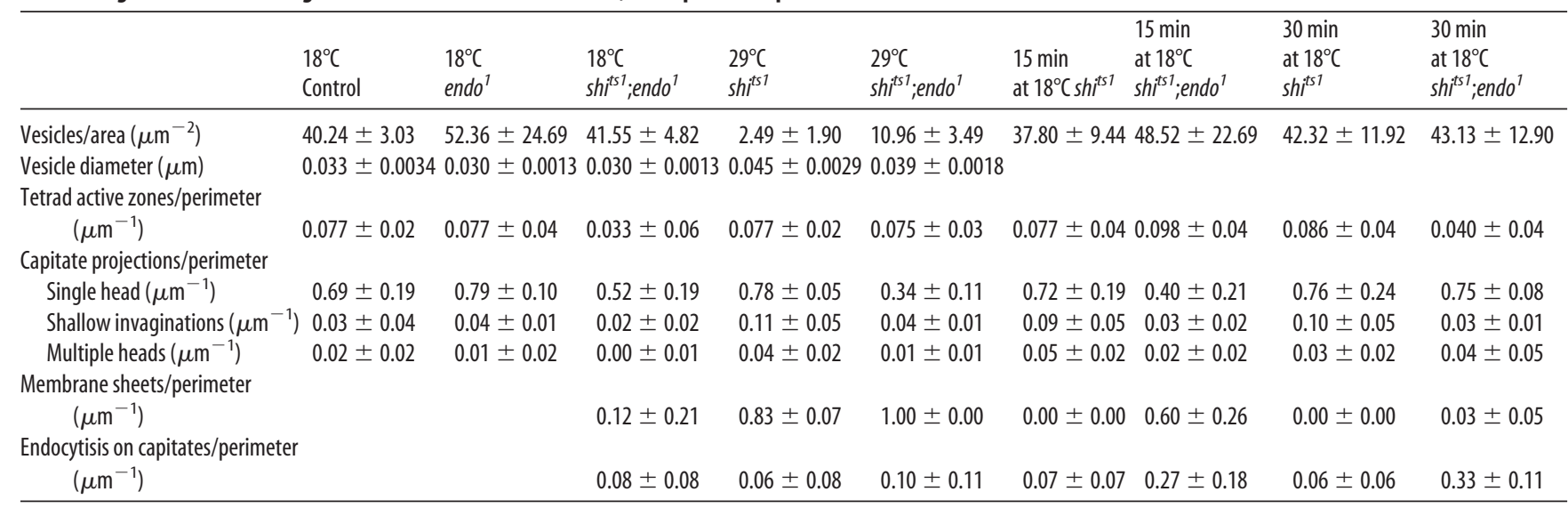

mostly to coincide with the immunogold localization of Endo in wild-type photoreceptor terminals, at capitate projections (Figs. $3,7 C, D)$. The size of the cluster and the normal proximity of capitate projections to the photoreceptor terminal synapses meant that all three were closely grouped. Although the distribution of vesicles in endo ${ }^{1}$ mutant terminals was altered, neither their total number nor their overall packing density in the cross section differed significantly from that in control terminals. Hence the distribution and appearance of synaptic vesicles is skewed in endo ${ }^{1}$ mutant photoreceptor terminals.

To test whether ultrastructural defects can also be observed when Endo protein levels are severely reduced but not absent, we created flies with homozygous mutant eyes for an endo hypomorphic allele, endo ${ }^{2}$ (Verstreken et al., 2002). Although we observed differences in the appearance and distribution of synaptic vesicles in endo ${ }^{1}$ null mutant photoreceptors, when we analyzed the ultrastructure of R1-R6 terminals in flies with homozygous mutant endo ${ }^{2}$ eyes no obvious vesicle phenotype was apparent, nor did we detect any other obvious ultrastructural defect. Similarly, hypomorphic alleles such as endo ${ }^{10}$ (Guichet et al., 2002) or endo ${ }^{2}$ (Verstreken et al., 2002) or partial rescue of the endo ${ }^{1}$ null allele by expressing wild-type Endo protein (Rikhy et al., 2002) all reveal much weaker phenotypes than endo null alleles at the NMJ. These data indicate that the complete removal of Endo is required to reveal clearly its function in vivo.

Given that Endo has been localized by immunogold in photoreceptor terminals to the capitate projections, we next considered the possibility that Endo may play a role in the biogenesis or invagination of capitate projections. From counts of all capitate projection profiles in control and endo ${ }^{1}$ mutant terminals, however, we found no statistically significant differences in either the number or size of capitate projections between control and endo ${ }^{1}$ mutant terminals (Table 1). Moreover, counts of active zones, the tetrad synapses (Meinertzhagen and O'Neil, 1991; Meinertzhagen and Sorra, 2001), in control and mutant terminals, also did not show a difference (Table 1). Thus, the loss of Endo function seems not to directly affect either the sites of exocytosis or the capitate projections.

\section{Epistatic relations between dynamin and Endophilin} Depletion-reformation assay

To study the function of Endo during synaptic vesicle recovery, we next created flies with endo ${ }^{1}$ mutant photoreceptors in which we could block the synaptic vesicle cycle temporarily, allowing us to deplete the synaptic terminals of functional, releasable vesicles.
To do this, we created eyes that were double-mutant for endo ${ }^{1}$ and $s h i^{t s 1}$. shits encodes a temperature-sensitive mutation in dynamin, which is essential for vesicle fission from the plasma membrane. At the restrictive temperature $\left(29^{\circ} \mathrm{C}\right)$ all synaptic vesicle recycling is blocked in $s h i^{t s 1}$, whereas at the permissive temperature $\left(18^{\circ} \mathrm{C}\right)$ endocytosis can proceed (Kosaka and Ikeda, 1983; Chen et al., 1991). Hence, when $s h{ }^{t s}{ }^{1}$; endo ${ }^{1}$ double-mutant terminals were brought to the restrictive temperature in the light $\left(15 \mathrm{~min}\right.$ at $\left.29^{\circ} \mathrm{C}\right)$, functional vesicles were depleted. When these depleted double-mutant $s h i^{t s 1}$; endo ${ }^{l}$ terminals were then brought back to the permissive temperature, we could follow the effects of the lack of Endo function during the recovery of the vesicles from endocytotic blockade (Fig. $8 \mathrm{~A}$ ). We monitored the recovery of these double-mutant terminals by EM after various times (light: 0,15 , or 30 $\mathrm{min}$ ) at the permissive temperature (Figs. 8-10). Data from doublemutant photoreceptor terminals were then compared with data from $s h i^{\text {tsl }}$ single-mutant terminals subjected to the same paradigm.

\section{Capitate projections proliferate during recovery from an} endocytotic block

As $s h i^{t s I} ;$ endo ${ }^{1}$ mutant photoreceptors recovered from exposure to the restrictive temperature, they exhibited altered numbers of capitate projections compared with $s h i^{t_{s l}}$ photoreceptor terminals. At the restrictive temperature, the number of capitate projections in double-mutant terminals was unchanged compared with those in $s h i^{\text {tsl }}$;endo ${ }^{1}$ control animals at the permissive temperature or with those in $s h i^{t s l}$ flies at the restrictive temperature (Fig. 9A). During a $30 \mathrm{~min}$ recovery phase at $18^{\circ} \mathrm{C}$, however, the number of capitate projections in $s h i^{\text {tsl }} ;$ endo ${ }^{1}$ terminals increased compared with $s h i^{t s 1}$ or $s h i^{t s l} ; e n d o^{1}$ terminals at the permissive temperature (Fig. 9A, Table 1). Thus, it appears that when endocytosis is induced by the release of functional vesicles in $s h i^{t s 1}$ terminals recovering from endocytotic blockade, the number of capitate projections also increases, either by an increased rate of invagination or by a decreased rate of evagination, thus supporting a role for capitate projections in synaptic endocytosis.

\section{Membrane uptake at synapses requires components of} clathrin-mediated endocytosis

At least two morphologically distinct pathways for presynaptic membrane recovery have been observed at photoreceptor terminals in Drosophila (Koenig and Ikeda, 1996). One occurs away from the active zone and likely represents classical clathrinmediated endocytosis. The other occurs close to the active zone and is marked morphologically by the appearance of membrane 

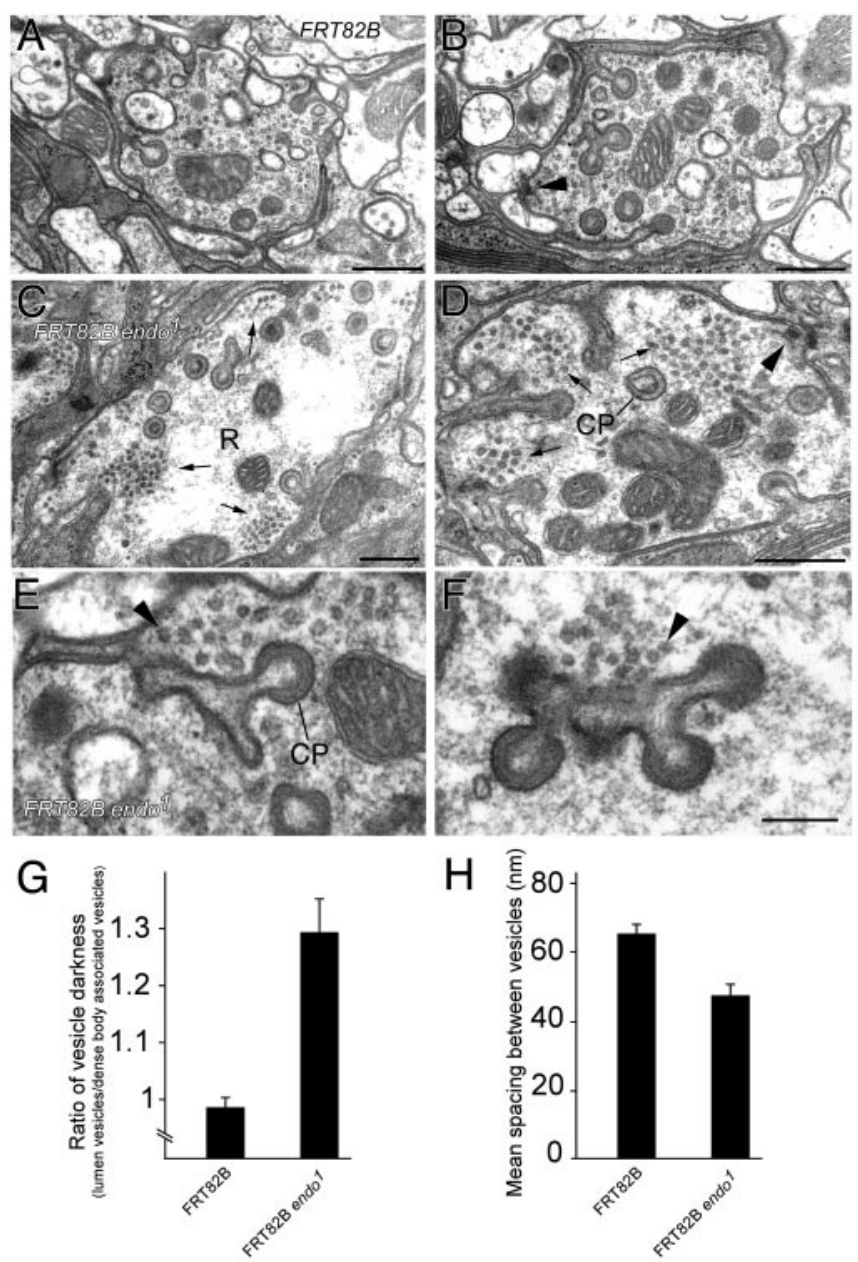

Figure 7. Synaptic vesicles cluster and are electron-dense in endo mutant terminals. $A-D$, EM images of R1-R6 terminal profiles ( $R$ in C). Scale bar, $0.5 \mu \mathrm{m} . A, B$, Control FRT $82 B$ terminals ( $y w$ eyFLP; FRT82B/FRT82B $C w^{+}{ }^{\text {) }}$. Synaptic vesicles appear more diffuse than in endo terminals ( $y w$ eyFLP; FRT82B/FRT82B $c l w^{+}$). C, D, Homozygous mutant FRT82B endo terminals. Synaptic vesicle clusters are marked by arrows. Note that clusters appear at or close to capitate projections ( $(P$ in $D)$, invaginations from the epithelial glia, and that tetrad synapses (arrowheads) also lie nearby. E, F, Enlarged view of electron-dense synaptic vesicle clusters (arrowheads) adjacent to profiles of capitate projections ( $(P)$, single-headed $(E)$ or multiple-headed $(F)$, in endo mutant terminals. Note the even spacing of vesicle profiles within cluster. Scale bar: (in $F) E, F, 0.2 \mu \mathrm{m}$. G, Normalized synaptic vesicle electron density, measured by calculating the ratio between the average pixel intensity of vesicles in clusters and the average pixel intensity of vesicles associated with the tetrad active zone $\left(n=15\right.$ terminals for both endo ${ }^{7}$ and control eyes). $H$, Mean nearest-neighbor spacing between vesicles in endo ${ }^{7}$ terminals and controls differs significantly; $p=0.0024$ ( $t$ test).

sheets that arise from the base of the T-shaped presynaptic ribbon. These represent the first sites of reinternalization of vesicle membrane, endocytotic invaginations of the plasmalemma ("cisternae") (Koenig and Ikeda, 1996) that fail to pinch off in $s h i^{t s l}$ held at the restrictive temperature. To determine the possible role of Endo in the uptake of membrane associated with the active zone, we counted the number of membrane sheets (Figs. $8 D$, inset, $9 B$ ) emerging from active zones in $s h i^{t s 1}$;endo ${ }^{1}$ and $s h i^{t s 1}$ control photoreceptor terminals after exposure to the restrictive temperature. In $s h i^{\text {tsl }} ;$ endo $^{l}$ double-mutant terminals, some membrane sheets already existed during exposure to the restrictive temperature (data not shown), and they persisted for a period of up to $30 \mathrm{~min}$ during the recovery at $18^{\circ} \mathrm{C}$ (Fig. $9 \mathrm{~B}$, Table $1)$. These data suggest that membrane uptake and vesicle refor- mation from such sheets at the active zone depend on the function of Endo and likely occur via a clathrin-mediated pathway.

Lack of Endophilin leads to accumulation of postfission vesicles

To determine which fraction of the vesicle pool in $s h i^{t_{s} 1}$;endo ${ }^{1}$ mutant R1-R6 terminals is functional, we exposed flies to $29^{\circ} \mathrm{C}$ for $15 \mathrm{~min}$ and assessed the extent of vesicle depletion (Table 1). Although $s h i^{t s l}$ terminals at the restrictive temperature were almost completely depleted of synaptic vesicles (Figs. 8 B, 9C), their depletion in $s h i^{t s l}$;endo ${ }^{1}$ terminals was much less severe, with surviving vesicles forming more densely packed clusters than in $s h i^{t s 1}$ single-mutant terminals (Figs. 8D, 9C). Their packing density in $s h i^{\text {tsl }}$;endo ${ }^{1}$ terminals at the restrictive temperature was in turn approximately four times less than in control terminals. The remaining vesicles in $s h i^{t s 1}$;endo ${ }^{1}$ double-mutant terminals exposed to high temperature, in addition to being clustered, also appeared electron dense (Fig. 8C), reminiscent of the clustered vesicles in endo ${ }^{1}$ single-mutant terminals (Figs. $7 C-F, 8 D, E$ ). Such dark vesicles were not observed in $s h i^{\text {ts }}$ at the restrictive temperature (Fig. $8 B, C)$ (data not shown) (Koenig and Ikeda, 1996). Hence, the lack of Endo in double-mutant terminals created a pool of vesicles that would normally be depleted by loss of shi function at restrictive temperatures. Apparently the vesicles that persist in $s h i^{t s l}$;endo ${ }^{1}$ terminals differed in some qualitative way from normal synaptic vesicles, not allowing them to reenter the synaptic vesicle cycle and be released at restrictive temperatures (Fig. $8 D, E)$.

Capitate projections are sites of endocytosis

To locate sites of endocytosis in the photoreceptor terminal, we examined in detail the occurrence of endocytotic structures in $s h i^{t s l}$ terminals during recovery from the restrictive temperature. We observed several late-stage omega structures and clathrincoated collared pits with or without short stalks that emerged from the plasma membrane. Interestingly, these structures quite often occurred along the stalks of capitate projections (Fig. $10 A, B$, Table 1 ). To test whether the formation of these endocytotic structures was blocked by additional removal of Endo function, we also examined flies with eyes double mutant for $s h i^{t s 1}$ and endo ${ }^{1}$ in our vesicle depletion-reformation assay. Significantly, during recovery from endocytotic blockade, endocytotic structures similar to those observed in $s h i^{t s l}$ recovering terminals were also observed in the double-mutant terminals, suggesting that Endo does not play a role in the formation of these endocytotic structures (Fig. 10C,D). Strikingly, however, these endocytotic structures again often occurred along the stalks of the capitate projections (Figs. $8 D$, inset, $10 C, D$ ). Together with the previous observations, these data indicate that capitate projections are sites of endocytosis in photoreceptor terminals (Fig. 11).

\section{Discussion}

During intense stimulation of a neuron, numerous synaptic vesicles fuse with the presynaptic membrane. To maintain synaptic transmission, neurons regenerate fusion-competent synaptic vesicles at the nerve terminal (Zhang and Ramaswami, 1999). Mechanisms of endocytotic retrieval have mostly been examined at the synapses of spiking neurons, such as mouse hippocampal neurons and the motor neurons of vertebrates and invertebrates such as Caenorhabditis elegans and Drosophila (Bellen, 1999). Much less attention has been given to vesicle retrieval mechanisms in nonspiking neurons (Roberts and Bush, 1981), such as Drosophila photoreceptors. In these neurons the quantity of neurotransmitter released is sensitive to small changes in presynaptic membrane potential (Rao-Mirotznik et al., 1995), and transmit- 
A

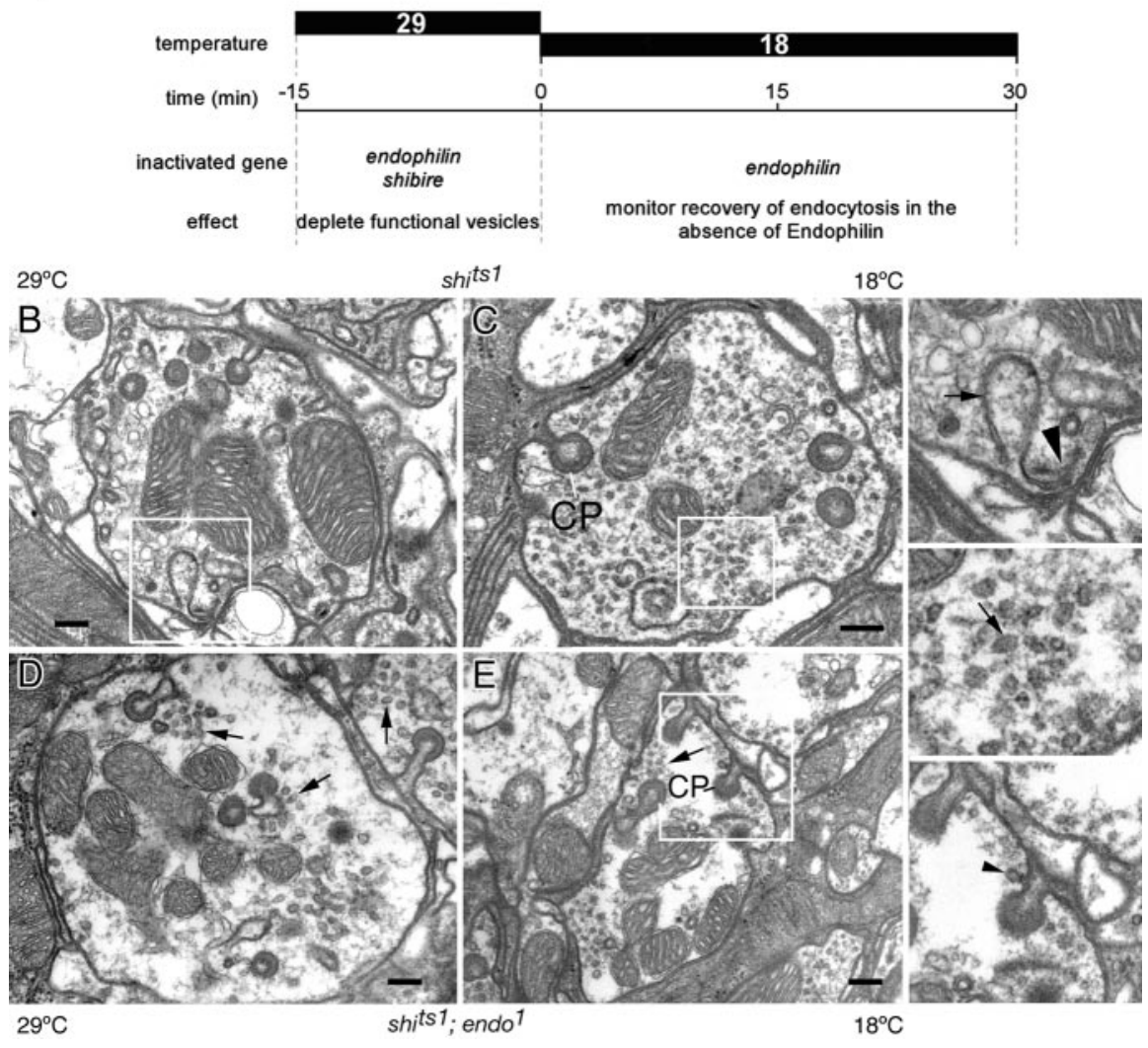

Figure 8. Vesicle reformation in $s i^{\text {ts }}$; endo ${ }^{1}$ double-mutant terminals. A, Experimental plan. Flies with shit ${ }^{\text {ts }}$; endo ${ }^{1}$ doublemutant and shit $^{\text {ts }}$ single-mutant eyes were shifted to the restrictive temperature for $15 \min (-15-0)$ to deplete synaptic vesicles (see Results for details and Materials and Methods for genotypes). They were then shifted back to the permissive temperature, revealing at 0,15 , and $30 \mathrm{~min}$ the surviving defects in endocytosis in the absence of Endo function, for comparison with terminals of $\mathrm{shi}^{\text {ts }}$; endo flies kept at the permissive temperature. $B-E$, Examples of terminals during recovery from exposure to the restrictive temperature in single-mutant $s i^{i t s}$ either dark-exposed at $29^{\circ} \mathrm{C}(B)$ or after 30 min recovery at $18^{\circ} \mathrm{C}$ from previous exposure to $29^{\circ} \mathrm{C}(\mathrm{C})$, and in double-mutant $\mathrm{sh}^{i t}{ }^{\mathrm{t}}$; ; endo ${ }^{1}$ either at $29^{\circ} \mathrm{C}(\mathrm{D})$ or $18^{\circ} \mathrm{C}$ after 30 min recovery from $29^{\circ} \mathrm{C}(E)$. Membrane sheet in $B$, enlarged in the top right-hand panel (arrow), arises from the pedestal of a presynaptic T-bar ribbon (arrowhead). Synaptic vesicle profiles (arrow) are enlarged from recovering shit ${ }^{\text {ts }}$ terminal $(C)$ in the middle right-hand panel, and an endocytotic profile (arrowhead) is enlarged from recovering shi ${ }^{\text {is }}$; endo ${ }^{7}$ terminal $(E)$ in the bottom right-hand panel.
A

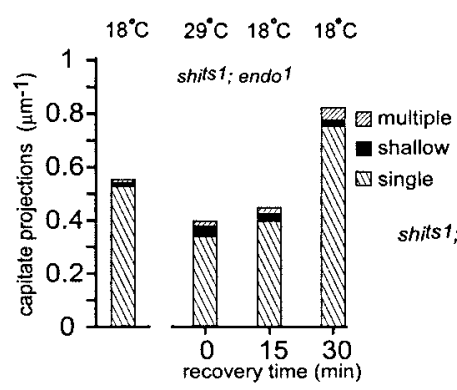

B

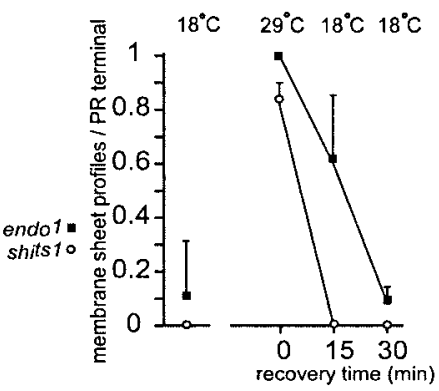

C

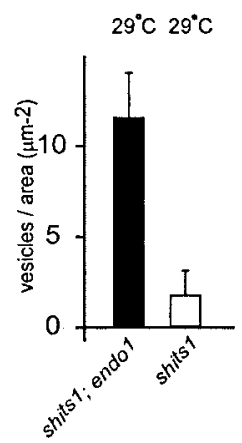

Figure 9. Time course of recovery from exposure to the restrictive temperature among organelle populations in $s h i^{\text {ts } 1}$ and $s i^{i t 5}$; endo ${ }^{1}$ terminals. A, During recovery from exposure to the restrictive temperature, the number of capitate projections in $\mathrm{shi}^{i t}{ }^{\text {; }}$; endo ${ }^{1}$ terminals increases; differences are significant after $15 \mathrm{~min}$ ( $p=0.036$; $t$ test) of recovery, but lose significance after $30 \mathrm{~min}$ ( $p=0.072$; $t$ test). We counted different morphological forms of capitate profiles (those with shallow, single, and multiple heads, which are thought to constitute the sequence for organelle formation and regression). $B$, Membrane sheets emerging from near the pedestal of the T-bar ribbon, probably representing endocytosis from that site (Koenig and Ikeda, 1996), persisted longer in shi ${ }^{\text {ts } 1}$; endo ${ }^{1}$ exposed to the restrictive temperature than in shits ${ }^{\text {t }}$ single-mutant terminals. C, More synaptic vesicles survive in

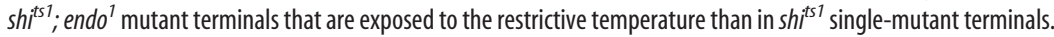

ter is released in a tonic manner (Juusola et al., 1996). Although the molecular mechanisms of endocytosis in the synaptic terminals of Drosophila photoreceptors have been studied in only a few mutants (Koenig and Ikeda, 1996; Littleton et al., 2001; Chang et al., 2002), the visual system in Drosophila is ideally suited to study such questions. Not only is it amenable to genetic manipulation through the creation of whole-eye mosaics (Stower and Schwarz, 1999; Newsome et al., 2000), but its modular organization is also well suited to detailed ultrastructural analyses (Meinertzhagen and O'Neil, 1991; Koenig and Ikeda, 1996; Meinertzhagen, 1996).

\section{Endo function is required to promote} the progression of newly internalized vesicles in the synaptic vesicle cycle Interfering with Endo function at live synapses has demonstrated the essential role of Endo during endocytosis of synaptic vesicles. Even so, the exact function of Endo remains controversial. Biochemical analyses and vesicle reformation assays have suggested a role for the protein during endocytosis via LPAAT activity, in the transition from shallow to deeply invaginated pits, possibly by modifying lipid structure (Scales and Scheller, 1999; Schmidt et al., 1999; Hill et al., 2001). Further support for a role of Endo during membrane bending has been suggested from analyses of endo hypomorphic alleles at the Drosophila NMJ and from injection experiments in the lamprey. At the Drosophila NMJ, although partial loss of endo function in hypomorphic alleles such as endo ${ }^{10}$ does not dramatically reduce total vesicle number, shallow pits accumulate along the plasma membrane (Guichet et al., 2002). Furthermore, when Endo antibodies, which probably disrupt some of the functions of Endo, are injected in tonically stimulated lamprey synapses, accumulations of shallow and more deeply invaginating coated pits are observed (Ringstad et al., 1999). These data are consistent with an early role of Endo in clathrin-mediated endocytosis. It is unlikely, however, that all endo function is completely removed in any of these assays, which may therefore not necessarily reveal the exact function of Endo during vesicle retrieval. The importance of LPAAT activity, in particular, remains controversial. Although Endo binds and tubulates membranes in vitro, this property is independent of the enzymatic activity of Endo (Farsad et al., 2001).

Other injection experiments suggest an additional function for Endo at the lam- 


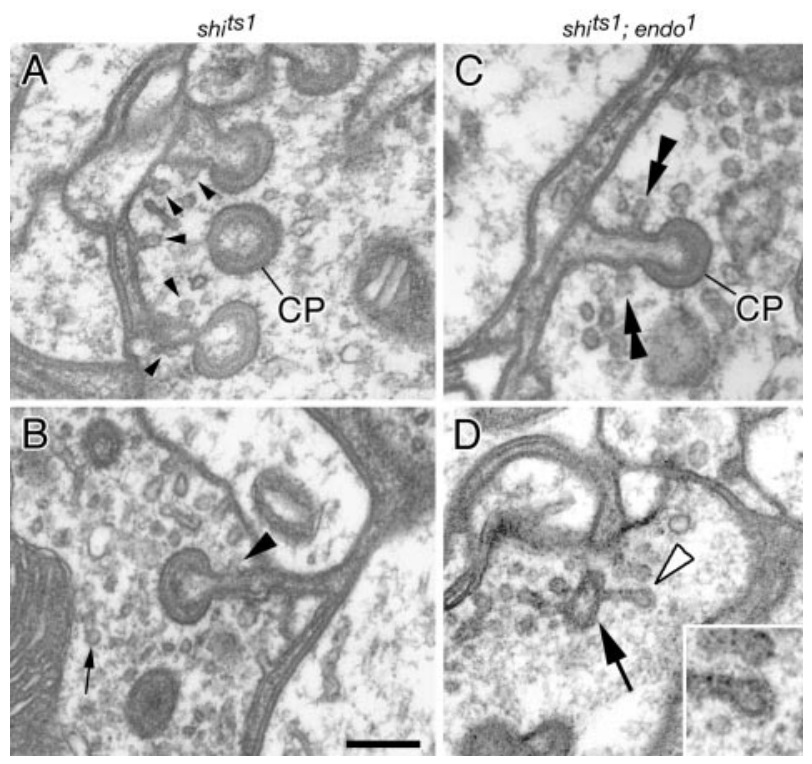

Figure 10. Endocytotic profiles form on the stalks of capitate projections in shit ${ }^{\text {ts } 1}$ mutant terminals recovering from exposure to the restrictive temperature. $A, B$, Profiles of new vesicles $\left(B\right.$, arrowhead) endocytosing from capitate projections $(A, C P)$ in terminals from shis ${ }^{\text {ts } 1}$ flies held previously at $29^{\circ} \mathrm{C}$ and then for $15 \mathrm{~min}$ at $18^{\circ} \mathrm{C}$. Endocytotic profiles appear along stalks as well as in nearby plasmalemma ( $A$, small arrowheads). C, D, Profiles of vesicles (double arrowheads) arising from stalks of capitate projections $(C, C P)$ in double-mutant shits ; endo terminals recovering from previous exposure to the restrictive temperature. Vesicles often arise from paired locations as seen in longitudinal ( () sections of the stalk. In a cross section of the stalk ( $D$, arrow), an extended endocytotic profile is surmounted by a vesicle (open arrowhead) that appears to be coated (inset). Note that free vesicles are electron-dense and that they persist during exposure of $\operatorname{sh}^{i t}{ }^{\text {i }}$; endo ${ }^{7}$ to the restrictive temperature, whereas in shit ${ }^{\text {is }}$ terminals $(C, D)$, vesicles do not remain coated (Koenig and lkeda, 1996). Scale bar, $0.2 \mu \mathrm{m}$.

prey synapse. When peptides that are thought to interfere with the $\mathrm{SH} 3$ domain interactions of Endo are injected into lamprey synapses, an accumulation of free, coated vesicles, with fewer earlier endocytotic intermediates, is observed (Gad et al., 2000). These data indicate that Endo functions at a late endocytotic step to promote vesicle uncoating; however, in these experiments only the Endo SH3 domain interactions were perturbed. Possible additional functions of Endo, for example those responsible for an early role in endocytosis, encoded by different parts of the protein, were probably left intact. As a result, the exact role of Endo in synaptic vesicle endocytosis remains unclear, because none of the reported assays removed all Endo function.

The data that we present here from an endo null mutant demonstrate that Endo is not essential during early endocytotic stages to bend and shape the synaptic membrane into a new vesicle. Rather, the data indicate that the function of Endo is required to promote the normal progression of newly internalized vesicles through the synaptic vesicle cycle. First, loss of Endo function compromises the synaptic-vesicle cycle, eliminating or reducing the on and off transients of the ERG (Rikhy et al., 2002). Second, ultrastructural analysis of endo ${ }^{1}$ mutant R1-R6 terminals reveals the accumulation of late endocytotic electron-dense vesicles, which appear in clusters. Third, when $s h i^{t s 1}$; endo ${ }^{1}$ terminals are shifted to restrictive temperatures in the light, significant numbers of nonfunctional vesicles remain and do not enter the synaptic vesicle cycle. Fourth, when $s h i^{t s l}$; endo ${ }^{1}$ terminals recover from endocytotic blockade at the permissive temperature, electron-dense vesicles bud from the membrane surrounding capitate projections, leading to the formation of the dark vesicle clusters. Such vesicle clusters are also observed in $s h i^{\text {tsl }}$ single-

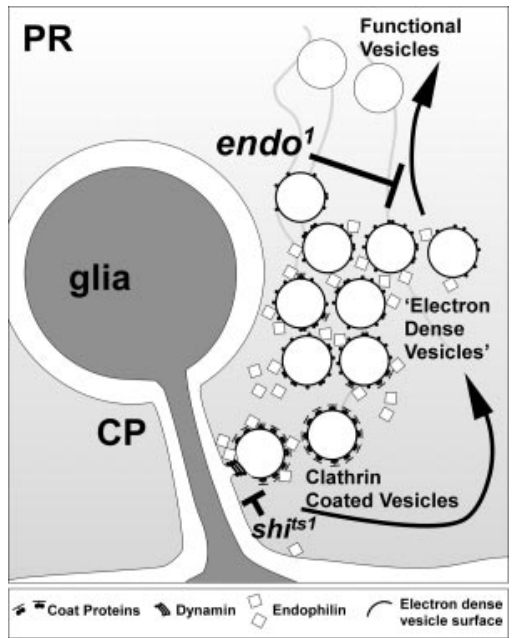

Figure 11. Endophilin is required for endocytosis, which occurs at glial invaginations in synaptic terminals of photoreceptors. Clathrin-coated vesicles can form on stalks of capitate projections (CP), which are invaginations of epithelial glia into the synaptic terminals of photoreceptor cells (PR). Newly endocytosed vesicles transform into electron-dense vesicles that are then recycled and pumped with neurotransmitter to become functional vesicles. The function of Endophilin is ultimately essential for newly internalized vesicles to progress into the synaptic vesicle cycle. Endo may be required to modify lipid composition to allow progression in the vesicle cycle or may be essential to recruit components of the uncoating machinery at an earlier stage of vesicle endocytosis.

mutant terminals in flies that are shifted from the restrictive temperature and allowed to recover at the permissive temperature (Koenig and Ikeda, 1999), although the vesicles in that case in $s h i^{t s l}$ are not electron dense. We therefore propose that in fly photoreceptor terminals Endo is not essential to depress the membrane into a deeply invaginating coated pit during early steps of endocytosis, nor does Endo appear essential for vesicle fission. Instead, our data chiefly support peptide injection experiments that interfere with Endo-SH3 function in lamprey synapses (Gad et al., 2000) and suggest that Endo function is ultimately required to promote the progression of newly internalized vesicles (Fig. 11). This idea is further supported by the ability of Endo to bind the protein Synaptojanin, which is also implicated in uncoating synaptic vesicles (Cremona et al., 1999; Nonet et al., 1999).

In summary, we find that Endo is not required for normal development of the Drosophila eye, a process that requires the interplay of numerous developmental pathways (Hsiung and Moses, 2002). In addition, the function of Endo is dispensable for axon outgrowth and target recognition. Endo is required, on the other hand, for synaptic vesicles to progress through the synaptic vesicle cycle. In addition, our data indicate that Endo performs this function at or around sites of glial invagination, which we propose are major centers for endocytosis in Drosophila photoreceptor terminals (Fig. 11.).

\section{Capitate projections are sites of clathrin-mediated endocytosis in photoreceptor terminals}

Our findings shed light on glial invaginations into photoreceptor terminals called capitate projections (Stark and Carlson, 1986). These are proposed synaptic organelles (Pyza and Meinertzhagen, 1997) and are known to be dynamic, capable of changing number within $\sim 10 \mathrm{~min}$ (Brandstätter and Meinertzhagen, 1995; Rybak and Meinertzhagen, 1997), but otherwise lack any reported function. Our observations now allow us to propose a particular role for these structures in clathrin-mediated endocy- 
tosis. First, immuno-EM with anti-Endo and anti-clathrin shows that both of these markers of clathrin-mediated endocytosis are enriched at vesicles and membranes associated with capitate projections. Second, in endo ${ }^{1}$ mutant photoreceptor terminals, electron-dense vesicles cluster near the capitate projections. Third, when clathrin-mediated endocytosis is blocked, the number of capitate projections per terminal increases. Finally, during recovery from endocytotic blockade in $s h i^{t s l}$; endo ${ }^{l}$ eyes, deeply invaginating coated pits are observed on the stalks of capitate projections. Hence, we propose that these glial invaginations, the capitate projections, play a prominent role in clathrin-mediated endocytotic vesicle retrieval (Fig. 11). It is therefore interesting that at the frog NMJ, endocytotic intermediates at the presynaptic membrane have also been observed in association with glial cell processes extending into the motor nerve terminal (Heuser and Reese, 1973).

Glia are also implicated in the clearance of transmitter from the synaptic cleft and in transmitter reuptake by the presynaptic terminal (Schousboe and Hertz, 1981; Steinhäuser and Gallo, 1996; Palmada and Centelles, 1998). Likewise, epithelial glia surrounding photoreceptor terminals in the lamina could be involved in transmitter re-uptake into the terminals. The epithelial glia express Ebony (Richardt et al., 2002), a major enzyme for the metabolism of released histamine (Borycz et al., 2002), which thus may clear transmitter from the cleft. We propose that in addition to their role in endocytosis, capitate projections may also aid in the transport of neurotransmitter back into the photoreceptor terminal, thereby linking at a single site the function of vesicle recovery from the membrane with transmitter recycling. In this context, it may be relevant that mutants of ebony and tan, which together regulate histamine metabolism (Borycz et al., 2002), have altered numbers of capitate projections (Meinertzhagen and Wang, 1997).

\section{Flies with endo ${ }^{1}$ mutant eyes can still see}

Although their on and off ERG transients are essentially absent, flies with endo ${ }^{1}$ mutant eyes are still not blind. Our evidence does not allow us to suggest that their vision is normal, only that phototaxis choice behavior under the conditions of our assay does not differ from controls. We have not examined the light intensity threshold for such behavior or the many other tests of visual behavior (Heisenberg and Wolf, 1984) that could be examined. It is therefore interesting that under different conditions and using a different genetic background, Rikhy et al. (2002) report that flies with endo ${ }^{1}$ mutant eyes, although not behaving as well as controls in a light-choice test, were also not entirely blind. Thus, our data as well as theirs indicate that at least some neurotransmitter release from endo ${ }^{1}$ photoreceptors must persist to give rise to signals in the lamina; even if these may be amplified at more central synapses in the visual pathway, they are sufficient to endow the flies with phototactic behavior. This persistent release must occur despite the loss of the ERG transients and implies that some regeneration of fusion-competent vesicles still proceeds in the absence of Endo function. Two mechanisms may exist to achieve this in endo ${ }^{l}$ mutant photoreceptors. Either residual endocytosis and minute rates of regenerating fusion-competent vesicles, sufficient for the fly to detect light, or alternative modes of synaptic vesicle regeneration that do not require Endo persist in endo ${ }^{1}$ mutant terminals. Given previous findings on endo ${ }^{1}$ mutant NMJs (Kjaerulff et al., 2002; Marte, 2002; Verstreken et al., 2002), it seems likely that in the fly's eye, too, a fast vesicle retrieval pathway operates to ensure the regeneration of fusion-competent vesicles.

\section{References}

Aravanis AM, Pyle JL, Tsien RW (2003) Single synaptic vesicles fusing transiently and successively without loss of identity. Nature 423:643-647.
Artalejo CR, Henley JR, McNiven MA, Palfrey HC (1995) Rapid endocytosis coupled to exocytosis in adrenal chromaffin cells involves $\mathrm{Ca}^{2+}$, GTP, and dynamin but not clathrin. Proc Natl Acad Sci USA 92:8328-8332.

Bellen HJ (1999) Neurotransmitter release. In: Frontiers in molecular biology, Vol 23 (Hames BD, Glover DM, eds), p 436. Oxford: Oxford UP.

Benzer S (1973) Genetic dissection of behavior. Sci Am 229:24-37.

Berdnik D, Torok T, González-Gaitán M, Knoblich JA (2002) The endocytic protein alpha-Adaptin is required for numb-mediated asymmetric cell division in Drosophila. Dev Cell 3:221-231.

Borycz J, Borycz JA, Loubani M, Meinertzhagen IA (2002) tan and ebony genes regulate a novel pathway for transmitter metabolism at fly photoreceptor terminals. J Neurosci 22:10549-10557.

Braitenberg V (1967) Patterns of projection in the visual system of the fly. I. Retina-lamina projections. Exp Brain Res 3:271-298.

Brandstätter JH, Meinertzhagen IA (1995) The rapid assembly of synaptic sites in photoreceptor terminals of the fly's optic lobe recovering from cold shock. Proc Natl Acad Sci USA 92:2677-2681.

Burg MG, Sarthy PV, Koliantz G, Pak WL (1993) Genetic and molecular indentification of a Drosophila histidine decarboxylase gene required in photoreceptor transmitter synthesis. EMBO J 12:911-919.

Ceccarelli B, Hurlbut WP, Mauro A (1973) Turnover of transmitter and synaptic vesicles at the frog neuromuscular junction. J Cell Biol 57:499-524.

Chang HC, Newmyer SL, Hull MJ, Ebersold M, Schmid SL, Mellman I (2002) Hsc70 is required for endocytosis and clathrin function in Drosophila. J Cell Biol 159:477-487.

Chen D, Stark WS (1993) Effects of temperature on visual receptors in temperature-sensitive paralytic shibire $\left(\operatorname{shi}^{\text {ts }}\right)$ mutants of Drosophila. J Insect Physiol 39:385-392.

Chen MS, Obar RA, Schroeder CC, Austin TW, Poodry CA, Wadsworth SC, Vallee RB (1991) Multiple forms of dynamin are encoded by shibire, a Drosophila gene involved in endocytosis. Nature 351:583-586.

Coombe PE (1986) The large monopolar cells L1 and L2 are responsible for ERG transients in Drosophila. J Comp Physiol [A] 159:655-665.

Cremona O, Di Paolo G, Wenk MR, Lüthi A, Kim WT, Takei K, Daniell L, Nemoto Y, Shears SB, Flavell RA, McCormick DA, De Camilli P (1999) Essential role of phosphoinositide metabolism in synaptic vesicle recycling. Cell 99:179-188.

Deitcher DL, Ueda A, Stewart BA, Burgess RW, Kidokoro Y, Schwarz TL (1998) Distinct requirements for evoked and spontaneous release of neurotransmitter are revealed by mutations in the Drosophila gene neuronalsynaptobrevin. J Neurosci 18:2028-2039.

Fabian-Fine R, Skehel P, Errington ML, Davies HA, Sher E, Stewart MG, Fine A (2001) Ultrastructural distribution of the $\alpha 7$ nicotinic acetylcholine receptor subunit in rat hippocampus. J Neurosci 21:7993-8003.

Farsad K, Ringstad N, Takei K, Floyd SR, Rose K, De Camilli P (2001) Generation of high curvature membranes mediated by direct endophilin bilayer interactions. J Cell Biol 155:193-200.

Fesce R, Meldolesi J (1999) Peeping at the vesicle kiss. Nat Cell Biol 1:E3-E4. Fischbach K-F, Dittrich APM (1989) The optic lobe of Drosophila melanogaster. I. A Golgi analysis of wild-type structure. Cell Tissue Res 258:441-475.

Gad H, Ringstad N, Löw P, Kjaerulff O, Gustafsson J, Wenk M, Di Paolo G, Nemoto Y, Crum J, Ellisman MH, De Camilli P, Shupliakov O, Brodin L (2000) Fission and uncoating of synaptic clathrin-coated vesicles are perturbed by disruption of interactions with the $\mathrm{SH} 3$ domain of endophilin. Neuron 27:301-312.

Gandhi SP, Stevens CF (2003) Three modes of synaptic vesicular recycling revealed by single-vesicle imaging. Nature 423:607-613.

González-Gaitán M, Jäckle H (1997) Role of Drosophila $\alpha$-adaptin in presynaptic vesicle recycling. Cell 88:767-776.

Guichet A, Wucherpfennig T, Dudu V, Etter S, Wilsch-Bräuniger M, Hellwig A, González-Gaitán M, Huttner WB, Schmidt AA (2002) Essential role of endophilin A in synaptic vesicle budding at the Drosophila neuromuscular junction. EMBO J 21:1661-1672.

Hardie RC (1987) Is histamine a neurotransmitter in insect photoreceptors? J Comp Physiol [A] 161:201-213.

Harrison SD, Broadie K, van de Goor J, Rubin GM (1994) Mutations in the Drosophila Rop gene suggest a function in general secretion and synaptic transmission. Neuron 13:555-566.

Heisenberg M (1971) Separation of receptor and lamina potentials in the electroretinogram of normal and mutant Drosophila. J Exp Biol 55:85-100.

Heisenberg M, Wolf R (1984) Vision in Drosophila. Berlin: Springer. 
Heuser JE, Reese TS (1973) Evidence for recycling of synaptic vesicle membrane during transmitter release at the frog neuromuscular junction. J Cell Biol 57:315-344.

Hiesinger PR, Reiter C, Schau H, Fischbach K-F (1999) Neuropil pattern formation and regulation of cell adhesion molecules in Drosophila optic lobe development depend on synaptobrevin. J Neurosci 19:7548-7556.

Hiesinger PR, Scholz M, Meinertzhagen IA, Fischbach K-F, Obermayer K (2001) Visualization of synaptic markers in the optic neuropils of Drosophila using a new constrained deconvolution method. J Comp Neurol 429:277-288.

Hill E, van Der Kaay J, Downes CP, Smythe E (2001) The role of dynamin and its binding partners in coated pit invagination and scission. J Cell Biol 152:309-324.

Homyk Jr T, Pye Q (1989) Some mutations affecting neural or muscular tissues alter the physiological components of the electroretinogram in Drosophila. J Neurogenet 5:37-48.

Hsiung F, Moses K (2002) Retinal development in Drosophila: specifying the first neuron. Hum Mol Genet 11:1207-1214.

Jarousse N, Kelly RB (2001) Endocytotic mechanisms in synapses. Curr Opin Cell Biol 13:461-469.

Juusola M, French AS, Uusitalo RO, Weckström M (1996) Information processing by graded-potential transmission through tonically active synapses. Trends Neurosci 19:292-297.

Kelly LE (1974) Temperature-sensitive mutations affecting the regenerative sodium channel in Drosophila melanogaster. Nature 248:166-168.

Kjaerulff O, Verstreken P, Bellen HJ (2002) Synaptic vesicle retrieval: still time for a kiss. Nat Cell Biol 4:E245-248.

Koenig JH, Ikeda K (1996) Synaptic vesicles have two distinct recycling pathways. J Cell Biol 135:797-808.

Koenig JH, Ikeda K (1999) Contribution of active zone subpopulation of vesicles to evoked and spontaneous release. J Neurophysiol 81:1495-1505.

Kosaka T, Ikeda K (1983) Possible temperature-dependent blockage of synaptic vesicle recycling induced by a single gene mutation in Drosophila. J Neurobiol 14:207-225.

Kral K, Meinertzhagen IA (1989) Anatomical plasticity of synapses in the lamina of the optic lobe of the fly. Philos Trans R Soc Lond B Biol Sci 323:155-183.

Littleton JT, Bai J, Vyas B, Desai R, Baltus AE, Garment MB, Carlson SD, Ganetzky B, Chapman ER (2001) synaptotagmin mutants reveal essential functions for the $\mathrm{C} 2 \mathrm{~B}$ domain in $\mathrm{Ca}^{2+}$-triggered fusion and recycling of synaptic vesicles in vivo. J Neurosci 21:1421-1433.

Marte B (2002) An encore for kiss and run? Nat Cell Biol 4:E123.

Meinertzhagen IA (1996) Ultrastructure and quantification of synapses in the insect nervous system. J Neurosci Methods 69:59-73.

Meinertzhagen IA, O'Neil SD (1991) Synaptic organization of columnar elements in the lamina of the wild type in Drosophila melanogaster. J Comp Neurol 305:232-263.

Meinertzhagen IA, Sorra KE (2001) Synaptic organization in the fly's optic lamina: few cells, many synapses and divergent microcircuits. Prog Brain Res 131:53-69.

Meinertzhagen IA, Wang Y (1997) Drosophila mutants tan and ebony have altered numbers of capitate projections, glial invaginations into photoreceptor terminals. In: Neurobiology: from membrane to mind, Vol II (Elsner N, Wässle H, eds), p 457. Stuttgart: Georg Thieme Verlag.

Newsome TP, Asling B, Dickson BJ (2000) Analysis of Drosophila photoreceptor axon guidance in eye-specific mosaics. Development 127:851-860.

Nonet ML, Holgado AM, Brewer F, Serpe CJ, Norbeck BA, Holleran J, Wei L, Hartwieg E, Jorgensen EM, Alfonso A (1999) UNC-11, a Caenorhabditis elegans AP180 homologue, regulates the size and protein composition of synaptic vesicles. Mol Biol Cell 10:2343-2360.

Palfrey HC, Artalejo CR (1998) Vesicle recycling revisited: rapid endocytosis may be the first step. Neuroscience 83:969-989.

Palmada M, Centelles JJ (1998) Excitatory amino acid neurotransmission. Pathways for metabolism, storage and reuptake of glutamate in brain. Front Biosci 3:D701-718.

Poodry CA, Hall L, Suzuki DT (1973) Developmental properties of Shibi$r e^{t s l}$ : a pleiotropic mutation affecting larval and adult locomotion and development. Dev Biol 32:373-386.

Pyza E, Meinertzhagen IA (1997) Circadian rhythms in screening pigment and invaginating organelles in photoreceptor terminals of the housefly's first optic neuropile. J Neurobiol 32:517-529.
Rao-Mirotznik R, Harkins AB, Buchsbaum G, Sterling P (1995) Mammalian rod terminal: architecture of a binary synapse. Neuron 14:561-569.

Richardt A, Rybak J, Störtkuhl KF, Meinertzhagen IA, Hovemann BT (2002) Ebony protein in the Drosophila nervous system: optic neuropile expression in glial cells. J Comp Neurol 452:93-102.

Rikhy R, Kumar V, Mittal R, Krishnan KS (2002) Endophilin is critically required for synapse formation and function in Drosophila melanogaster. J Neurosci 22:7478-7484.

Ringstad N, Gad H, Löw P, Di Paolo G, Brodin L, Shupliakov O, De Camilli P (1999) Endophilin/SH3p4 is required for the transition from early to late stages in clathrin-mediated synaptic vesicle endocytosis. Neuron 24:143-154.

Roberts A, Bush BMH (1981) Neurones without impulses: their significance for vertebrate and invertebrate nervous systems. Cambridge, UK: Cambridge UP.

Rybak J, Meinertzhagen IA (1997) The effects of light reversals on photoreceptor synaptogenesis in the fly Musca domestica. Eur J Neurosci 9:319-333.

Sanyal S, Tolar LA, Pallanck L, Krishnan KS (2001) Genetic interaction between shibire and comatose mutations in Drosophila suggest a role for snap-receptor complex assembly and disassembly for maintenance of synaptic vesicle cycling. Neurosci Lett 311:21-24.

Scales SJ, Scheller RH (1999) Lipid membranes shape up. Nature 401:123-124.

Schmidt A, Wolde M, Thiele C, Fest W, Kratzin H, Podtelejnikov AV, Witke W, Huttner WB, Soling H-D (1999) Endophilin I mediates synaptic vesicle formation by transfer of arachidonate to lysophosphatidic acid. Nature 401:133-141.

Schousboe A, Hertz L (1981) Role of astroglial cells in glutamate homeostasis. Adv Biochem Psychopharmacol 27:103-113.

Schulze KL, Broadie K, Perin MS, Bellen HJ (1995) Genetic and electrophysiological studies of Drosophila syntaxin-1A demonstrate its role in nonneuronal secretion and neurotransmission. Cell 80:311-320.

Simpson F, Hussain NK, Qualmann B, Kelly RB, Kay BK, McPherson PS, Schmid SL (1999) SH3-domain-containing proteins function at distinct steps in clathrin-coated vesicle formation. Nat Cell Biol 1:119-124.

Slepnev VI, De Camilli P (2000) Accessory factors in clathrin-dependent synaptic vesicle endocytosis. Nat Rev Neurosci 1:161-172.

Somogyi P, Takagi H (1982) A note on the use of picric acidparaformaldehyde-glutaraldehyde fixative for correlated light and electron microscopic immunocytochemistry. Neuroscience 7:1779-1783.

Stark WS, Carlson SD (1986) Ultrastructure of capitate projections in the optic neuropil of Diptera. Cell Tissue Res 246:481-486.

Steinhäuser C, Gallo V (1996) News on glutamate receptors in glial cells. Trends Neurosci 19:339-345.

Stower RS, Schwarz TL (1999) A genetic method for generating Drosophila eyes composed exclusively of mitotic clones of a single genotype. Genetics 152:1631-1639.

Sun J-Y, Wu X-S, Wu L-G (2002) Single and multiple vesicle fusion induce different rates of endocytosis at a central synapse. Nature 417:555-559.

Trujillo-Cenóz O (1965) Some aspects of the structural organization of the intermediate retina of dipterans. J Ultrastruct Res 13:1-33.

Verstreken P, Kjaerulff O, Lloyd TE, Atkinson R, Zhou Y, Meinertzhagen IA, Bellen HJ (2002) Endophilin mutations block clathrin-mediated endocytosis but not neurotransmitter release. Cell 109:101-112.

Wu MN, Fergestad T, Lloyd TE, He Y, Broadie K, Bellen HJ (1999) Syntaxin $1 \mathrm{~A}$ interacts with multiple exocytic proteins to regulate neurotransmitter release in vivo. Neuron 23:593-605.

Zakharenko SS, Zablow L, Siegelbaum SA (2002) Altered presynaptic vesicle release and cycling during mGluR-dependent LTD. Neuron 35:1099-1110.

Zhang B, Ramaswami M (1999) Synaptic vesicle endocytosis and recycling. In: Neurotransmitter release, Vol 23 (Bellen HJ, ed), p 436. Oxford, UK: Oxford UP.

Zhang B, Koh YH, Beckstead RB, Budnik V, Ganetzky B, Bellen HJ (1998) Synaptic vesicle size and number are regulated by a clathrin adaptor protein required for endocytosis. Neuron 21:1465-1475.

Zinsmaier KE, Eberle KK, Buchner E, Walter N, Benzer S (1994) Paralysis and early death in cysteine string protein mutants of Drosophila. Science 263:977-980.

Zipursky SL, Venkatesh TR, Teplow DB, Benzer S (1984) Neuronal development in the Drosophila retina: monoclonal antibodies as molecular probes. Cell 36:15-26. 